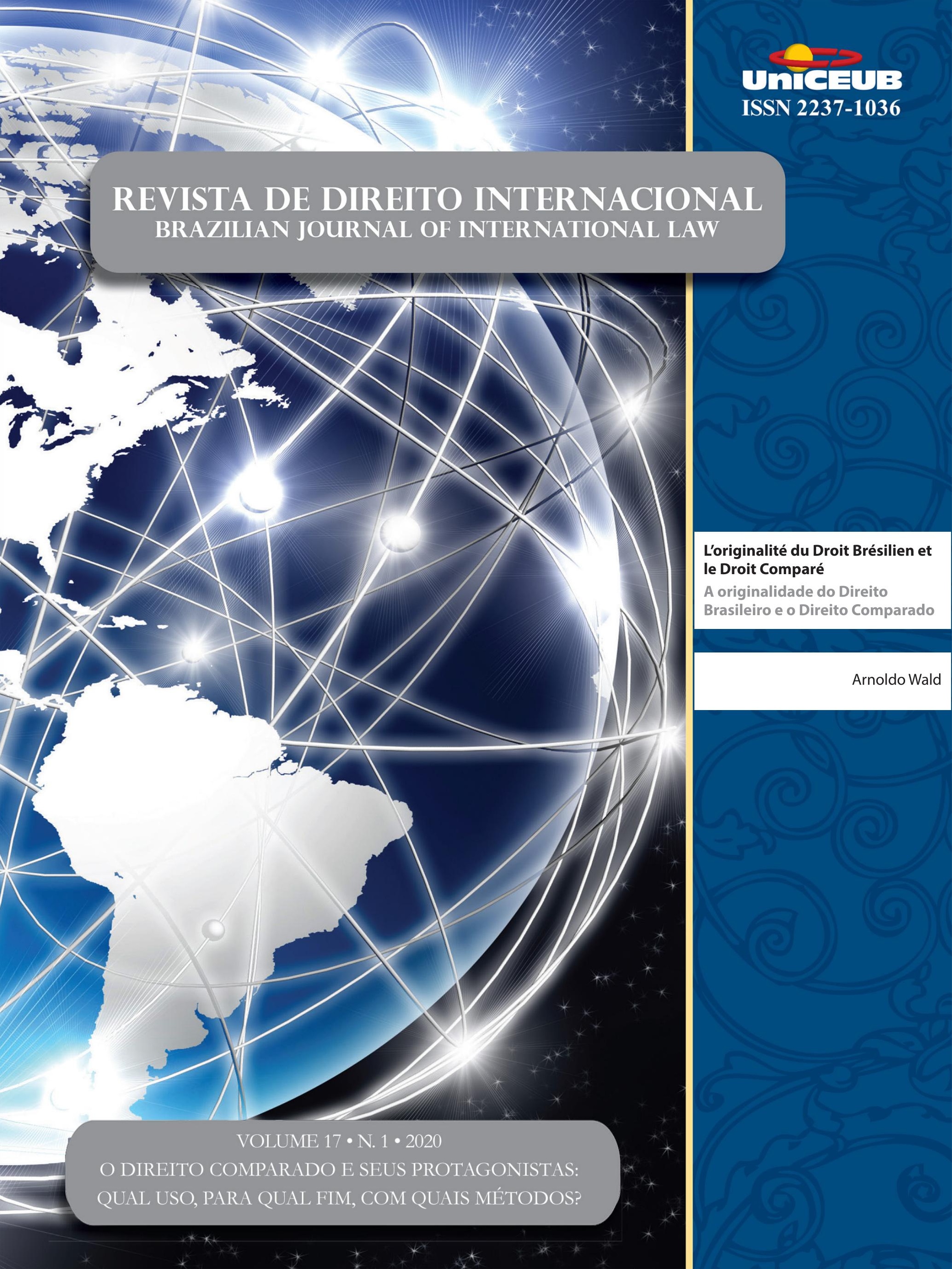


Parte I - O Direito comparado e seus Protagonistas: QuAL uso, PARA QUAL FIM, COM QUAIS MÉTODOS? .....................................................................1

EDITORIAL ............................................................................................................... 3

"Tudo o que precisamos fazer é ter certeza de que continuaremos conversando".............................. 3

Gustavo Cerqueira e Patrícia Perrone Campos Mello

Direito comparado E METOdologia $\quad$................................................... 6

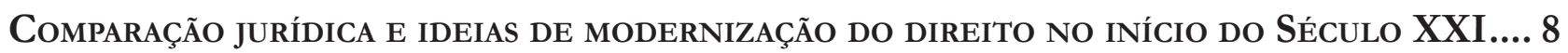
Gustavo Cerqueira

O DIREITO COMPARADO: ESFORÇO DE RESGATE HISTORIOGRÁFICO E DE PROBLEMAS METODOLÓGI$\cos$

Arnaldo Sampaio de Moraes Godoy e Gustavo Fereira Ribeiro

Direito Comparado e Política: Reflexões Necessárias .42

Raphael Carvalho de Vasconcelos e Deo Campos Dutra

Direito comparado no Brasil

L'originalité du Droit Brésilien et le Droit Comparé .57

Arnoldo Wald

LEI DA BOA RAZÃo E COMPARATISMO JURÍDICO NA DOUTRINA CIVILISTA BRASILEIRA DE 1850 A 1880

Alan Wruck Garcia Rangel

O STF EM REDE? QUANTO, COMO, COM QUE ENGAJAMENTO ARGUMENTATIVO O STF USA PRECEDENTES ESTRANGEIROS EM SUAS DECISÕES?

Patrícia Perrone Campos Mello e Felipe Meneses Graça 
Suprema Imprecisão: a metodologia em Direito Constitucional Comparado E as deficiênCias Em Seu uso pelo Supremo Tribunal Federal

Alonso Freire e Hugo Sauaia

Direitos comparados

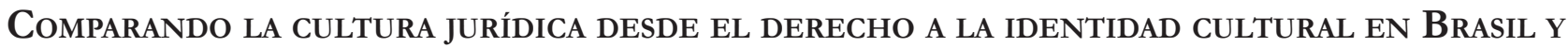

CHILE 145 Juan Jorge Faundes e Fabian Le Bonniec

O Divino e o Racional no Direito: notas para um diálogo entre sistemas jurídicos 181 Salem Hikmat Nasser e José Garcez Ghirardi

Regulação do discurso de Ódio: análise comparada em países do Sul Global 196 Jane Reis Gonçalves Pereira, Renan Medeiros de Oliveira e Carolina Saud Coutinho

Parte II - Outros temas

LA OTRA CARA DE LA MONEDA: PROTECCIÓN CONSTITUCIONAL DE LA EMPRESA, EL EMPRENDIMIENTO Y LA LIBRE COMPETENCIA EN CHILE y COLOMBiA

Juan Pablo Díaz Fuenzalida e Juan Sebastián Villamil Rodríguez

The European Court of Human Rights Decision on there 'Burqa Ban'and the CritiCal analysis of the Pragmatic experimental logic 258 Flavianne Fernanda Bitencourt Nóbrega e George Browne Rego

Direitos humanos das deslocadas ambientais e os impactos da Usina de Belo Monte: da EXPLORAÇÃO AMAZÔNICA À SUBJUGAÇÃO FEMININA

Thais Silveira Pertille e Letícia Albuquerque

Solução de Controvérsias em Acordos de Investimento: as experiênCias do CPTPP, CETA E DOS ACFIs

Fábio Morosini, Vivian Daniele Rocha Gabriel e Anastacia Costa

50 anos dos “direitos da Criança” na Convenção Americana de Direitos Humanos: a HISTÓRIA DO ARTIGO 19 311

Sven Peterke e Paloma Leite Diniz Farias 
EMPRESAS E DIREITOS HUMANOS: COMPARTILHANDO VALOR E RESPONSABILIDADES .325 Melina Girardi Fachin

CONTEMPORARY RESPONSES TO BUSINESSES' NEGATIVE HUMAN RIGHTS IMPACT 341 Andres Felipe Lopez

Human Right to LABOR PROTECTION IN UKRAINE: CURRENT SITUATION AND THE PROSPECTS OF IMPLEMENTATION OF INTERNATIONAL RULES 363 Nina Daraganova

International Regulation and Global Governance: The EU influential Method IN TIMES OF NORMATIVITY CHANGE 373 Gabriela Hühne Porto, Paula Wojcikiewicz Almeida e Juliana Maia F. A. Netto

Tecnologias digitais e o comércio de bens E SERviços na OMC/Digital. 391 Umberto Celli Junior

Los fuertes hacen lo QUe PUeden: exponiendo los límites de la Corte Penal InternaCIONAL .406

Cristián D. González-Ruiz e Víctor M. Mijares

Human Rights, Humanitarian Law and State Power 418 Renata Nagamine e João Roriz

Regional integration in the South Pacific: Challenges for Public governance .433 Joanna Siekiera

Parte III - Resenhas 443

RESENHA DA OBRA

“Democracia e policentrismo do poder", de Murilo Gaspardo 445 Angela Limongi Alvarenga Alves 


\title{
L'originalité du Droit Brésilien et le Droit Comparé*
}

\section{A originalidade do Direito Brasileiro e o Direito Comparado}

\begin{abstract}
Arnoldo Wald**
Les droits des pays latins d'Amérique, faits pour s'appliquer dans un milieu différent du milieu américain, se distinguent des droits de l'Europe continentale et constituent, au sein du groupe du droit français, une catégorie originale, dont les traits spécifiques apparaissent de plus en plus clairement au fur et à mesure que le divorce s'atténue entre des textes jadis théoriques et les réalités de la vie. ${ }^{1}$

[...] des leçons doivent, de ce côté-ci du globe, être tirées de l'expérience juridique brésilienne, soit dans certains détails de sa législation, soit - et c'est sans aucun doute le plus important - dans l'attitude d'héritière inventive qui caractérise son évolution, et que l'on gagnerait à répandre en France. 2

[...] Le droit brésilien se caractérise en effet par la vitesse des changements accomplis car il s'agit d'un pays en voie de développement rapide où le législateur non seulement accompagne les faits mais les devance souvent avec des textes qui sont élaborés pour accélérer le développement économique et social. ${ }^{3}$
\end{abstract}

\section{Résumé}

Cet article examine le rôle du Droit Comparé au Brésil, ainsi que le degré de son influence sur sa formation en tant qu'ordre juridique autonome et l'étendue de son impact et diffusion au sein de l'enseignement et de la pratique des avocats et juges du pays.

Mots Clé: Droit Comparé. Objet. Métode. Droit brésilien. Influence

\section{Resumo}

O presente artigo examina o papel do Direito Comparado no Brasil, tanto quanto o grau da sua influência na formação deste como ordem jurídica autônoma e a extensão do seu impacto e difusão no ensino e na praxe dos advogados e juízes do país.

* Auteur invité / Guest Author / Autor convidado

** Avocat aux barreaux de São Paulo et Paris. Professeur titulaire de droit civil à l'Université de l'Etat de Rio de Janeiro. Docteur Honoris Causa de l'Université de Paris II, Président d'Honneur du Groupe brésilien de l'Association Henri Capitant. Email: aw@wald.com.br

\footnotetext{
René DAVID, Traité élémentaire de Droit Civil Comparé, Paris, LGDJ, 1950, p. 267.

2 Guy Canivet, Tirer profit du droit brésilien. In: Le droit brésilien d'hier, d'aujourd'hui et de demain, Dir. par Arnoldo Wald et Camille Jauffret-Spinosi, Paris, Société de Législation Comparée, 2005, p. 13.

3 Bénédicte Fauvarque-Cosson, Le droit brésilien, cet “inconnu” qui ne l'est plus. In: A evolução do direito no século XXI: estudos em homenagem ao Prof. Arnoldo Wald, Coord. Diogo Leite de Campos, Gilmar Ferreira Mendes, Ives Gandra da Silva Martins, Coimbra, Almedina, 2007, p. 36.
} 
Palavras-Chave: Direito Comparado. Objeto. Método. Direito brasileiro. Influência

\section{Introduction}

Etant donnée la connaissance très relative et parfois assez superficielle que les juristes étrangers ont du Brésil et du droit brésilien, de son enseignement et de sa pratique, il nous parait utile de faire précéder notre article de quelques informations de base, qui faciliteront sa compréhension et permettront peut-être qu'il soit plus utile.

Il est d'ailleurs opportun de rappeler que, jusqu'à maintenant, la majorité des comparatistes, sauf quelques rares et importantes exceptions ${ }^{4}$, n'a pas approfondi l'étude des pays en développement et de leurs particularités. Cette absence de bibliographie juridique s'explique tant par des barrières linguistiques que par le fait que, jusqu'à maintenant, la présence des représentants de ces pays dans les séminaires et congrès internationaux a pendant de longues années été assez limitée.

La compréhension du droit des pays en développement exigeait donc une présence sur place, la connaissance de la langue locale et l'étude de la pratique, ce qui n'était pas toujours facile et commode. Il faut également signaler que les études de droit comparé, tant en Europe qu'aux Etats Unis, ont progressé en partant de l'analyse comparative entre des pays de même niveau économique et souvent du même régime politique pour, peu à peu, atteindre des législations d'autres Etats, en donnant préférence à ceux de plus grande importance dans le monde contemporain, comme cela a été le cas de l'URSS, de la Chine et de l'Inde.

En réalité, la comparaison entre les droits des pays développés et ceux des Etats en développement accéléré - que l'on peut désigner comme constituant le deuxième monde - et des autres nations oblige le juriste à un énorme effort pluridisciplinaire pour comprendre non seulement les différences des textes législatifs, de la jurisprudence et de la doctrine, mais aussi faire toute une analyse économique, historique et sociologique. Cette réalité est tellement importante qu'il nous parait même possible d'admettre, à coté des distinctions tra-

Parmi les comparatistes qui ont étudié le droit brésilien en profondeur, ayant vécu au Brésil, il faut citer les noms des professeurs René David et Tullio Ascarelli. ditionnelles entre les grands systèmes juridiques, une classification des droits nationaux selon le niveau de développement économique et culturel de chaque pays.

Or, parmi les pays en développement, il est certain que le Brésil présente un intérêt spécial en vertu tant de ses dimensions géographiques, démographiques et économiques, que du fait qu'il s'agit du plus grand Etat possédant ces particularités tout en étant lié à la culture occidentale et à son échelle de valeurs, au contraire de ce qui arrive dans les cas de la Chine et de l'Inde.

Dans une phase de mondialisation progressive, le Brésil, la principale puissance économique de l'Amérique Latine, représente également le centre du bloc du Mercosul à un moment où il cherche à consolider ses liens avec l'Union Européenne.

Ainsi, dans la mesure où le droit comparé est un catalyseur du rapprochement entre les peuples et de la meilleure connaissance de l'étranger, l'an 2000 se présente certainement comme étant le début du siècle de l'institutionnalisation de la comparaison juridique entre tous les pays du monde, même ceux en développement, au même titre que l'année 1900 a représenté la naissance d'une conception scientifique du droit comparé et en a défini la fonction, la méthode et les aspirations.

\section{Le Brésil, son droit et son monde juridique}

Une des dix premières économies du monde, avec un produit interne brut d'environ 1,8 billion de dollars, et une population de plus de 200 millions d'habitants, le Brésil qui se caractérise par la différence de niveau entre sa région centre et sud, d'un coté, et nord et nord-est de l'autre, rappelle un peu la situation de l'Italie à l'envers. ${ }^{5}$

C'est un professeur français, M. Jacques Lambert, qui a signalé avec beaucoup d'acuité l'existence de deux "Brésil" avec des phases de développement historique, économique et social très distinctes et un des nos sociologues a remarqué que le Brésil, par ces contrastes, est une "espèce de musée de l'histoire sociale", présentant simultanément des aspects de civilisation correspondant à des époques différentes.

Cette situation de fait rend difficilement applicables les mêmes normes juridiques à des populations si hétérogènes et oblige les juges à utiliser l'interprétation comme moyen d'adaptation de la loi aux conditions locales de vie, créant ainsi d'importantes divergences dans notre jurisprudence.

En outre, les lois brésiliennes, élaborées par une élite en contact avec les derniers progrès de la technique législative étrangère, ne sont pas 
Cette dualité du pays a été analysée par des sociologues brésiliens et français ${ }^{6}$.

Il faut également indiquer l'abîme qui a longtemps existé entre une législation faite par les élites, en se basant sur les règles constitutionnelles et les lois européennes ou américaines, et les sentiments et les besoins de la majorité de la population. ${ }^{7}$

Le droit public brésilien s'est surtout inspiré du droit américain, l'actuelle Constitution de 1988 ayant toutefois incorporé de nombreuses règles des traditions juridiques européennes, surtout dans le domaine économique et social. En droit privé, le Brésil se rattache plutôt au système du droit français, le Code Civil, qui est entré en vigueur en 2003, ayant également été profondément influencé par le droit italien. En droit pénal et en droit de la procédure, le Brésil a souffert une importante influence italienne et allemande, tandis que le droit commercial, dans sa législation la plus récente, a suivi le modèle des Etats-Unis.

Le Brésil a actuellement plus de 1.000.000 avocats ${ }^{8}$, ce qui représente un peu moins que ceux de l'Union Européenne et la moitié de ceux des Etats Unis, mais

toujours - comme l'a remarqué le Professeur René David - suffisamment basées sur la réalité brésilienne et correspondent souvent davantage à des conquêtes récentes dans les pays les plus développés qu'à des revendications réelles des milieux brésiliens. On a pu dire ainsi que nos lois étaient souvent simplement "programmatiques", c'est-à-dire valaient comme des directives ou des principes généraux que le législateur aimerait voir appliqués par le juge selon les possibilités et les conditions des différents milieux. » (Arnoldo WALD, La réforme du droit brésilien, Revue internationale de droit comparé, $\mathrm{n}$. 4, 1962, p. 713).

6 Ces affirmations faites il y a de longues années continuent à être actuelles. Jacques Lambert reconnait que le Brésil reproduit les contrastes du monde puisqu'on y retrouve des aspects qui rappellent New York ou Chicago à côté d'autres qui évoquent ceux de l'Inde et de l'Egypte (Le Brésil, Armand Colin, Paris, 1953, p. 64). Le sociologue français est revenu à ce sujet dans d'autres de ses ouvrages parmi lesquels son livre en portugais intitulé Os Dois Brasis (Les deux Brésils), qui a mérité de nombreuses rééditions et dans lequel il fait la différence entre le Brésil archaïque et le Brésil moderne, 12. ${ }^{a}$ ed., Cia Editora Nacional, São Paulo, 1984. La question a également été étudiée par Charles Moraze dans son ouvrage Les trois âges du Brésil, et par Roger Bastide dans Brésil, terre des contrastes. Il existe à ce sujet une très importante bibliographie brésilienne dont on peut mentionner, comme exemples, les travaux de Victor Nunes Leal, Coronelismo, enxada e voto, Gilberto Freyre, Casa Grande \& Senzala, Raymundo Faoro. Os donos do Poder et Sérgio Buarque de Holanda, Raízes do Brasil.

René DAVID. Traité élémentaire de droit civil compare. Paris, LGDJ, 1950 , p. 266.

8 La majorité des avocats inscrits au Barreau a toutefois d'autres occupations ou exerce la profession simultanément avec d'autres activités, ce que la loi brésilienne permet. quand même un dixième de la totalité des professionnels du monde entier. D'autre part, le pays a environ 15.000 juges, comprenant toutes les instances de la Justice des Etats, la Justice Fédérale et celle du travail. La Cour Suprême reçoit environ 40.000 recours par an et le Tribunal Supérieur de Justice, dernière instance en matière infra-constitutionnelle, apprécie près de 100.000 procès chaque année.

Le Tribunal de l'Etat de São Paulo a actuellement en cours environ plus de 10 millions de procédures, la plus grande partie étant représentée par ceux de l'administration contre le contribuable ou vice-versa. Une réforme judiciaire est en train d'être discutée au Congrès et l'on reconnaît que l'évolution économique récente a provoqué un certain retard du droit sur les faits, qui se fait sentir tant dans la formation des avocats et des juges que dans l'exécution de la justice.

Il y a, d'autre part, une inflation législative et de la réglementation que le gouvernement tâche de juguler, en donnant une nouvelle structure au système, par des consolidations, afin d'éviter une plus grande insécurité juridique.

Finalement, au moment de l'ouverture de l'économie brésilienne, qui jusqu’à 1990 était plutôt fermée, avec une grande ingérence de l'Etat et une protection excessive du marché national, les techniques juridiques et les règles de droit sont également en phase de révision. Ainsi, économistes et juristes discutent quel modèle doit adopter le Brésil, celui de la common law ou celui du droit continental d'origine romano-germanique ${ }^{9}{ }^{10}$. C'est dans ce contexte qu'il faut comprendre l'analyse qui suit.

\section{3 la signification et l'objet du Droit Comparé}

Le débat classique, relatif à la reconnaissance de l'autonomie scientifique du droit comparé ou à l'inverse,

9 Rudi DORNBUSCH. "Vérifier la législation avant d'investir ", article publié dans Business Week et reproduit par le quotidien brésilien. Gazeta Mercantil le 28 août 1996.

10 Dans ce contexte, il convient de mentionner la récente adoption progressive par le Droit brésilien des instituts ou de concepts juridiques inspirés par le common law, tels que la technique des décisions des Cours supérieures ayant effet contraignant por les juges des instances primaires, inspiré par le «stare decisis». 
à la considération de ce dernier en tant que simple méthode de recherche, agite, depuis longtemps, la doctrine, mais l'adhésion à cette seconde opinion n'implique en aucune manière la dévalorisation des travaux objets du droit comparé. La majorité de la doctrine brésilienne reconnaît le caractère scientifique du droit comparé. Science pour les uns et méthode pour les autres, selon le Professeur Dourado de Gusmão, le droit comparé serait à la fois une méthode et une science:

[...] en tant que méthode spécifique par rapport à celle adoptée par d'autres branches de la science juridique dont l'objet est le droit national, il permet une sorte d'investigation du droit. En tant que science, il envisage l'étude des différents systèmes de droit, afin d'identifier leurs affinités et leurs contrastes. ${ }^{11}$

Cette position n'est pas sans rappeler celle de H.C. Gutteridge, pour qui le droit comparé constituait une branche autonome parmi les sciences juridiques, dont l'objet serait l'étude, par la méthode comparative, des normes issues d'au moins deux systèmes juridiques. Pour bien comprendre ce raisonnement, il faut néanmoins revenir sur le concept de science juridique, en s'interrogeant afin de savoir si le droit comparé correspond à cette définition et, ensuite, pour tenter d'en déterminer l'objet.

Plus récemment Catherine Valcke tente de reconstruire le droit comparé comme une discipline ayant pour but non pas l'harmonisation juridique, ni même de promouvoir la diversité juridique à travers le monde, mais de comprendre chaque système juridique en soi selon ses propres caractéristiques et à la manière dont les acteurs de chaque système le font.

Cet auteur ajoute que la contribution des comparatistes se distingue de celle des juristes nationaux en ce sens qu'elle offre une compréhension interne de plusieurs ensembles de droit en même temps par des mouvements de flux et de reflux, en oscillant entre les perspectives interne et externe ${ }^{12}$.

\footnotetext{
11 Paulo Dourado de GUSMÃO. «Direito Comparado, sua realidade e suas utopias ». Revista Forense, v. 152, 1954. p. 20. Voir, du même auteur, "Filosofia do direito e direito comparado ». Revista Forense, v. 146, 1953. p. 3.

12 Cahterine Valcke. Comparationg law: compartive law as reconstruction of collective commitments. Cambridge: Cambridge University Perss, 2018.
}

\subsection{La nature scientifique du droit comparé}

Les méfiances et résistances rencontrées lorsque l'on affirme le caractère scientifique du droit trouvent, sans doute, leurs origines dans le concept de science élaboré par Aristote, pour qui une connaissance ne mérite pas d'être classée parmi les sciences si elle ne se fonde pas sur l'existence de «lois» établies à partir de la répétition de certains faits, ceux-ci constituant son objet, et d'une généralité qui n'existe pas dans le domaine des sciences sociales.

Plus récemment, le centre d'intérêt des philosophes dans la caractérisation d'une science n'a plus porté exclusivement sur les attributs de son objet, pour finalement s'orienter vers l'appréciation de sa méthodologie. Dans ce sens, l'extraordinaire influence de Hans Kelsen a été de la plus grande importance au Brésil, ses ouvrages étant aujourd'hui souvent consultés dans nos facultés de droit.

Par sa pureté méthodologique, Kelsen commence par établir la distinction entre les sciences naturelles, soumises à un principe de causalité, et les sciences normatives, soumises à l'imputation. La distinction s'obtient par le recours à deux catégories irréductibles: être et devoir-être (sein und sollen). Ainsi, les normes ne cherchent pas à décrire la conduite humaine telle qu'elle est ou telle qu'elle le sera, mais telle qu'elles doivent $t^{13}$. Au lieu de décrire la conduite, la norme de droit lui donne une signification, c'est à dire un sens juridique. Cela étant, les normes juridiques ne se soumettent pas à un critère de vérité, comme il en est des sciences causales, mais de simple validité. L'homme de science doit donc étudier le sens attribué à la conduite par la norme juridique en vigueur dans une collectivité.

Dans la formulation de ses postulats, la science du droit essaie de décrire les significatifs juridiques imputés à la conduite par les normes. Comme l'a déjà remarqué Lourival Vilanova ${ }^{14}$, il existe un double niveau de langage: un langage descriptif ou language-moyen, propre à la science juridique, chargé de décrire et de nous faire comprendre l'ordre juridique positif, et un langage de prescription de la conduite humaine, propre aux nor-

\footnotetext{
13 Hans KELSEN. Teoria Pura do Direito, São Paulo, Martins Fontes, 1998, p. 4.

14 Lourival VILANOVA, apud, Ivo DANTAS. «Direito comparado como ciência ». Revista de Informação Legislativa, Brasília, n.134, 1997. p. 231.
} 
mes appartenant à un système juridique donné.

Or, le fait que les chercheurs du droit comparé construisent leurs postulats à partir de l'analyse d'au moins deux ordres ou systèmes de droit différents n'enlève rien au caractère scientifique de ces activités. Au contraire, cela tend à rendre la recherche plus difficile car le juriste n'aura plus un seul mais deux ordres juridiques comme base empirique pour la confrontation de ses postulats. Allant dans le même sens, cet élargissement du champ de référence des constructions du droit comparé par rapport aux branches du droit national lui confère sa spécificité et sa légitimité. Cette dernière découlerait alors, tout simplement, de l'utilisation de la méthode comparative dans l'étude du droit comparé.

\subsection{L'objet du droit comparé}

La réalisation de certaines distinctions méthodologiques, afin de délimiter précisément l'objet des recherches, est nécessaire pour l'étude de toute science. Ainsi, suivant l'intention du chercheur, la comparaison peut s'appliquer à deux systèmes juridiques distincts, chacun d'eux renvoyant à des ordres juridictionnels distincts; mais peut aussi confronter deux ordres juridiques ou, également, des institutions juridiques déterminées, des branches du droit, des législations, etc. Il paraît donc possible de distinguer entre ce que les juristes brésiliens dénomment la macrocomparaison et la microcomparaison.

Selon l'idée macrocomparative, l'analyse des ordres juridiques devrait être faite à partir de la comparaison entre les systèmes juridiques dans lesquels ils s'incluent. A cet effet, la classification des systèmes élaborée par René David est la plus répandue au Brésil: la famille romano-germanique, celle des pays socialistes, celle dite $\mathrm{du}$ common-law, les systèmes confessionnels dans lesquels s'incluent les droits musulmans, le système indien de l'Extrême Orient et le système africain. ${ }^{15}$

D'autre part, dans une perspective de microcomparaison, le juriste peut se contenter de la comparaison entre deux ordres juridiques, étatiques ou pas, ou même limiter l'objet de sa confrontation à une institution juridique déterminée. Ainsi, dans un même pays à statut confessionnel, le juriste aura la tâche de comparer, par example, le mariage musulman et le mariage chrétien.

15 Lino MORAIS LEME, Direito Civil Comparado. São Paulo: Revista dos Tribunais, 1962.
L'objet des recherches peut également se réduire ou s'élargir selon la conception du droit retenue par chaque juriste. Comme son analyse descriptive doit porter sur le droit positif en vigueur dans au moins deux collectivités, une conception plus étroite du droit positif peut réduire de façon significative le domaine de la recherche.

Depuis la fin du XIXe siècle, la question est discutée par les juristes brésiliens. Clóvis Beviláqua, considéré comme l'un des plus importants comparatistes brésiliens ${ }^{16}$, a publié, en 1893, la première édition de ses $L i$ ções de Legislação Comparada, en démontrant l'importance de la comparaison comme technique de connaissance, compréhension et analyse du droit national et étranger qui justifie l'existence d'une science autonome. ${ }^{17} \mathrm{Il}$ s'oppose à l'époque à la thèse d'un autre professeur brésilien de procédure civile qui voyait, dans le droit comparé, l'instrument adéquat pour atteindre l'unification universelle du droit. ${ }^{18}$

Écrivant en 1903, le Professeur et ancien magistrat Candido de Oliveira résume son enseignement à la $\mathrm{Fa}$ culté de Droit de Rio de Janeiro dans son livre Curso de Legislação Comparada, en signalant l'importance du droit comparé dans l'élaboration législative comme une espèce de phare qui illumine le juriste dans son travail incessant d'adaptation de la loi aux nouvelles nécessités sociales. Il invoque également l’importance de l'école historique de Savigny et du Volksgeist, qui justifie l'évolution constante du droit, et finit par reconnaitre que, de la même façon que le commerce enrichit les peuples, les échanges des connaissances juridiques facilitent la révision de la législation, en inspirant tant la construction législative et jurisprudentielle que les recherches de la doctrine. Ainsi, à la vieille d'une nouvelle codification brésilienne (le Code Civil), Candido de Oliveira, qui ne connaissait pas encore les travaux du Congrès de Paris de 1900, évolue déjà par rapport à la position de Beviláqua. ${ }^{19}$

16 Kurt H. NADELMAN le cite comme le juriste brésilien le plus important pour le droit comparé en même temps que Teixeira de Freitas, Studi in memoria di Tullio Ascarelli, in Great Comparative Lawyers, Milan, Giuffrè, 1969, 3eme v. p. 1410.

17 Clóvis BEVILAQUA, Lições de Legislação Comparada. 2. ed. Bahia: Ed. José Luiz da Fonseca Magalhães, 1897. p. 12.

18 João MONTEIRO, A Universalização do Direito. São Paulo: [s.n.7, 1982.

19 Candido de Oliveira, Curso de Legislação Comparada. Rio de Janeiro: Jacinto Ribeiro dos Santos Editor, 1903. p. 6. Dans sa bibliographie l'auteur ne cite pas les travaux de Esmein, Saleilles ou Josserand, mais fait référence au Bulletin de la Société de législation comparée. 
Pendant la première moitié du XXe siècle, les juristes brésiliens ont reconnu que le droit comparé est «la science de notre temps». Ainsi le juge et Professeur Virgílio de Sá Pereira reprend, en 1931, dans son enseignement de doctorat à la Faculté de Droit de Rio de Janeiro, les thèses du Congrès de Droit Comparé de Paris et, en particulier, transmet à ses élèves les positions des comparatistes et civilistes français. ${ }^{20}$

Un troisième facteur d'importance dans la délimitation de l'objet de la science du droit comparé réside dans l'examen des sources du droit. L'objet du droit comparé étant l'analyse d'une pluralité d'ordres juridiques positifs et la notion de positivité étant inséparable de celle de source du droit, la base des recherches comparatives peut donc varier selon que l'on retient une notion large ou restreinte des sources du droit.

En somme, l'objet des sciences juridiques étant le droit positif, la détermination de l'objet d'étude du droit comparé variera selon la conception du droit positif adoptée, a priori, par le juriste. De la même façon, les bases empiriques de sa recherche s'élargiront ou se réduiront selon qu'il procède à une analyse macrocomparative ou microcomparative. En tout état de cause, parmi d'autres applications possibles, le résultat des recherches en droit comparé reste indéniablement utile à la compréhension du droit de la collectivité, d'où l'importance du droit comparé dans la formation des juristes.

On peut ainsi affirmer que, pour la doctrine brésilienne, comme pour un courant de la pensée juridique italienne, le droit comparé a comme fonction la connaissance plus approfondie des donnés juridiques, la promotion du meilleur modèle légal ou interprétatif ayant une grande importance mais n'étant qu'un but éventuel de la recherche du comparatiste. ${ }^{21}$

D'autre part, les juristes brésiliens sont également très sensibles aux enseignements d'André Tunc, quand il recommande que les cours de droit comparé portent autant sur la société que sur le droit, devant examiner les lois et les institutions présentes et passées, mais en ne négligeant pas leur avenir et, en particulier, les recher-

\footnotetext{
20 Fortunato AZULAY indique cette évolution selon laquelle les juristes ne se limitent plus à citer les lois étrangères mais aussi les instituts de chacun des pays, en introduisant des éléments historiques et sociologiques, In: Os Fundamentos do Direito Comparado. Rio de Janeiro: Edição da Empresa a Noite. p. 8.

${ }_{21}$ Rodolfo SACCO, La comparaison juridique au service de la connaissance du droit. Paris: Economica, 1991. p. 10.
}

ches qui présentent un intérêt pratique et social. ${ }^{22}$

Finalement, le juriste brésilien est aussi attentif à ce que la doctrine allemande a appelé le droit comparé de la post-modernité, qui explique non seulement les différences mais aussi les points communs du droit. Dans cette conception, il est très important d'examiner les techniques et les instituts en évolution en acceptant le pluralisme comme valeur juridique. ${ }^{23}$

\section{Le Droit Comparé dans la formation et les réformes du Droit Brésilien}

En raison de l'histoire de leur pays, les juristes d'Amérique Latine sont tous des comparatistes. Là est leur richesse, là a été aussi peut être leur faiblesse.

Richesse car la culture, et pas seulement la culture juridique des élites sud américaines, a toujours été impressionnante pour les Européens. En effet, l'apport espagnol ou portugais, puis l'apport français, puis nord-américain, et en même temps souvent aussi allemand ou italien, ont contraint les juristes d'Amérique latine à s'intéresser à d'autres droits qu'au leur, puisque leur propre droit émanait le plus souvent d'une grande variété d'inspirations étrangères. Ils devaient donc, pour comprendre leur propre droit, dépasser celui ci et s'intéresser aux droits étrangers.

C'est une grande opposition avec les juristes européens qui, bien trop souvent, n'étaient pas comparatistes et ne s'intéressaient qu'à leur propre système.

Mais faiblesse aussi, car pendant longtemps on a pu penser que les droits d'Amérique latine n'étaient pas des droits véritablement originaux, que leur système de droit était fait d'emprunts aux systèmes de droit européen, et que même souvent ces systèmes n'étaient qu'une adaptation plus ou moins servile

22 André TUNC, Jalons - Dits et écrits, publication de la Société de législation comparée, 1991, p. 69 et 80. Dans le même sens, voir Ignacio WINIZKY, De la technique comparative en droit, in Problèmes contemporains de droit comparé, Tome II, Institut Japonais de Droit Comparé, Université Chuo, 1962. Cet illustre professeur argentin a rappelé que, selon René DAVID, celui qui se consacre au droit comparé doit partager l'existence des peuples dont il examine le droit et ainsi connaitre l'essence de leur âme. Après quoi le Professeur Winizki conclut que la recherche comparative requiert comme condition de son existence, la vie en commun et la connaissance réelle de l'essence humaine des individus et des collectivités. Et il ajoute "c'est un moyen de réalisation de l'amour entre les peuples, s'il est vrai que connaître... c'est aimer" (op. cit. p. 534).

23 Jayme ERIK, Visões para uma teoria pós-moderna do direito comparado, traduction brésilienne d'une conférence prononcée à Osnabruck, le 5.6.1997, publiée par la Revista dos Tribunais, n. 759, p. 25. 
d'un système européen imité.

Mais, en 1953, René David dans un article, dénonçait de façon très pertinente cette façon de voir les choses et démontrait l'originalité des différents droits des pays d'Amérique du Sud. ${ }^{24}$

Le droit brésilien mérite d'être étudié pour l'intérêt propre que présente le droit de ce grand pays où des traditions qui sont les nôtres doivent être conciliées avec des donnés géographiques et sociales toutes différentes de celles de l'Ancien Monde: il est plein d'intérêt d'étudier les effets de ce heurt entre des traditions que l'on veut conserver et des réalités que l'on doit subir. ${ }^{25}$

La formation du droit brésilien a subi l'influence de modèles distincts. Tandis que le modèle juridique originaire des pays romano-germaniques a principalement inspiré la formation des institutions liées au droit privé, l'adoption d'institutions du droit public américain est également marquante, surtout à partir de la Constitution de 1891, la première des constitutions du Brésil républicain. Si un tel clivage entre un droit public soumis à l'influence nord-américaine et un droit privé inspiré du modèle juridique romano-germanique a été la principale clé à la disposition de ceux qui voulaient comprendre l'importance du droit comparé au Brésil, cette dichotomie a été légèrement altérée durant la dernière moitié du XXème siècle. Actuellement, nous constatons, au Brésil, une influence américaine croissante en ce qui concerne le droit privé, alors que dans les branches traditionnellement classées parmi celles du droit public, comme la concurrence et le droit administratif, ainsi qu'en matière de procédure civile, les juristes brésiliens se révèlent de plus en plus inclinés à s’inspirer des sources françaises et italiennes.

Après avoir constaté le caractère scientifique du droit comparé, il nous semble qu'une analyse de son influence sur la formation du droit brésilien ne peut se limiter à la seule étude de la réception des principes juridiques étrangers. Cette importation de modèles pouvant être due à plusieurs facteurs, elle ne dérivera du droit comparé que si elle est le résultat d'un effort scientifique de rationalisation. Il faut que cette influence découle de la recherche comparatiste, en ayant pour but de faire pro-

\footnotetext{
24 Camille Jauffret-Spinosi, «La circulation du modèle juridique français ", rapport des journées franco-italiennes de l'Association Henri Capitant de 1993, Travaux de l'Association, Tome XLIV. Paris: Litec, 1994. p. 109.

25 René David, «Le droit brésilien jusqu'en 1950 », In: Le droit brésilien d'hier, d'aujourd'hui et de demain, Arnoldo Wald et Camille JauffretSpinosi, (Dir.). Paris: Société de Législation Comparée, 2005. p. 26.
}

fiter le droit brésilien de l'expérience juridique d'autres peuples.

Dans ce sens, la pratique du droit comparé et son influence sur la formation du droit brésilien remonte à l'indépendance du pays, en 1822. Aucune norme brésilienne, en tant que telle, n'existant à l'époque, le nouveau souverain a prolongé l'application des anciennes règles d'origine portugaise qui sont restées en vigueur, dans la plupart des cas, pendant presque un siècle. N'ayant pas leur propre littérature, les juristes brésiliens furent, dès le début, des comparatistes par nécessité. L'absence d'une bibliographie nationale les obligeaient à faire du droit comparé par manque d'une doctrine et d'une jurisprudence nationales, à l'instar de Mr. Jourdain, qui utilisait la prose et les vers sans le savoir.

Ce contexte d'application de la doctrine étrangère n'allait guère changer jusqu'à la fin du XIX siècle et, en un certain sens, jusqu'à la fin de la première moitié du XXe siècle, malgré la publication de certains ouvrages de droit brésilien dont il faut reconnaitre l'importance. En 1889, le Brésil est devenu une République largement inspirée du modèle fédéraliste nord-américain. Un autre événement de la plus grande importance a été la promulgation, en 1917, du Code Civil brésilien, fruit lui aussi d'une recherche comparative, et celle du nouveau Code en 2002.

D'autre part, à partir de la fin de la Deuxiéme Guerre Mondiale nous avons eu un grand développement de la législation, de la jurispurdence et de la doctrine.

C'est ce que reconnait le Professeur Bénédicte Fauvarque-Cosson qui a présidé la Société de Législation Comparé et est maintenant au Conseil d'Etat. Daus un article intitulé « Le droit brésilien, cet « inconnu » que ne l'est plus » elle écrit:

D'un point de vue historique, ce qui frappe surtout, c'est ce renversement de perspective: tandis que pendant des siècles on a pu observer le rayonnement du Code civil français au Brésil et plus généralement en Amérique latine, le droit brésilien est en voie de devenir un modèle, non seulement en Amérique latine, mais aussi dans le monde et plus spécifiquement pour les juristes français et européens.

S'il fut d'abord l'héritier du droit de l'Europe continentale puis du droit public des Etats-Unis, ce droit brésilien se présente désormais comme un droit mixte, doté de ses caractéristiques propres. En effet, il a su concilier, de manière originale, la famille 
romano-germanique et celle de common law. ${ }^{26}$

Et elle ajoute:

[...] le Brésil montre que la loi et surtout la codification, constituent des instruments efficaces de développement économique, qui ne sont synonymes ni de rigueur, ni de lenteur. Le droit brésilien se caractérise en effet par la vitesse des changements accomplis car il s'agit d'un pays en voie de développement rapide où le législateur non seulement accompagne les faits mais les devance souvent " avec des textes qui sont élaborés pour accélérer le développement économique et social.

L'ancien Code civil, élaboré à la fin du XIXe siècle, datait de 1917. Le nouveau Code civil, qui intègre le droit commercial, a été promulgué en 2002. Original par sa conception et sa modernité, ce codes 'efforce de " concilier les traditions locales et nouvelles techniques, l'excès d'individualisme de la législation antérieure et les nécessités actuelles de la société brésilienne».

D'importantes modifications ont été accomplies, notamment en droit des contrats où l'on peut observer un véritable changement de la vision du contrat et la percée d'un courant proche de celui défendu par une partie de la doctrine française, connu en France sous le nom de « solidarisme contractuel.

Pour diverses raisons, le droit brésilien devrait, à l'avenir, inspirer les juristes français. Pour des raisons économiques, car les relations entre nos pays s'intensifient, « avec des investissements dans différents domaines, un nombre croissant de joint ventures et l'ouverture du marché des concessions et des PPP, qui intéressent les entreprises françaises $» .^{27}$

Il est intéressant de remarquer que dans les cours et monographies de droit comparé, les pays en développement n'ont, en général, pas été l'objet de nombreuses études, malgré un effort récent de publier un certain nombre de thèses des étudiants étrangers qui viennent se spécialiser en France et traitent avec des professeurs français des sujets qui leur permettent de profiter des connaissances qu'ils ont de leur droit national, en comparaison avec le droit français ${ }^{28}$.

Il est donc étonnant que, tandis qu'en économie, il y a des nombreux travaux en ce qui concerne les pays du tiers monde, le droit comparé, pendant très long-

\footnotetext{
26 In: A evolução do direito no século XXI: estudos em homenagem ao Prof. Arnoldo Wald. Coimbra: Almedina, 2007. p. 35.

27 In: A evolução do direito no século XXI, ..., p. 36.

28 C'est le cas du livre dirigé et publié en Belgique par Michel FROMONT et republié au Brésil avec le titre "Direito francês e direito brasileiro - perspectivas nacionais e comparadas ». São Paulo: IDP / Saraiva, 2017.
}

temps, ne s'en est pas occupé surtout en ce qui concerne l'Amérique Latine.

La mondialisation toutefois permis, récemment, une réaction constructive en ce domaine, en s'inspirant des leçons déjà citées du Professeur René David au sujet de l'intérêt que les juristes peuvent avoir d'étudier les problèmes et les solutions des pays neufs.

En 2001, ainsi, Vernon Valentine Palmer a publié son livre intitulé « Mixed Jurisdiction Worldwide - The Third Legal Family $»^{29}$, qui étudie les droits mixtes dans sept États : l'Écosse, le Québec, la Louisiane, l'Afrique du Sud, Porto Rico, les Philippines et Israël représentant au total 150 millions d'habitants. Il s'agit de législations qui n'ont pas une importance mondiale et qui peuvent être considérées comme des droits périphériques. Dans la précieuse note bibliographique qu'il fait à ce livre, le Professeur Blanc-Jouvan souligne toute son importance de ce travail et même son caractère pionnier, affirmant:

L'intérêt, considérable, d'un tel travail réside naturellement dans sa dimension comparative: car, pour la première fois, il ne s'agit pas seulement d'une comparaison "verticale ", qui tend à rapprocher chaque système étudié du modèle, de «droit civil» ou de common law, avec lequel il présente le plus d'affinités, mais d'une véritable comparaison " horizontale ", infiniment plus fructueuse, qui amène à confronter ces systèmes entre eux. Et la thèse avancée par l'auteur est que, malgré des différences évidentes (qu'il n'est pas question de sous-estimer), cette confrontation fait apparaitre suffisamment de similitudes pour que l'on puisse regrouper l'ensemble des droits mixtes (au sens précédemment indiqué) dans une catégorie unique, au point même d'y voir, à côté des deux familles traditionnelles, une «troisième famille juridique. $\|^{30}$

Le caractère constructif de cette symbiose entre les influences du droit civil et de la common law a également un effet enrichissant pour la science juridique, car comme l'affirme encore le Professeur Blanc-JOUVAN:

Chemin faisant, nous découvrons que le métissage
ainsi intervenu entre deux grandes traditions
juridiques a entraîné, dans la doctrine comme chez
les praticiens, une créativité plus grande et fait
apparaître, grâce à de subtiles combinaisons, des
règles et des institutions nouvelles qui vont bien
au-delà de ce qui résulterait normalement d'une
simple transplantation (comme en matière de trust,
d'estoppel, de cause, etc). Il y a là un facteur non

29 Vernon Valentine Palmer, Mixed Jurisdiction Worldwide - The Third Legal Family. Cambridge: Cambridge University Press, 2001, XVI. 496 p.

30 Xavier Blanc-Jouvan, op. cit., p. 891. 


$$
\text { négligeable de progrès du droit. }{ }^{31}
$$

Lors des commémorations du bicentenaire du Code Napoléon, nous avons assisté à une espèce de résurrection ou, au moins de renouvellement, du droit comparé en vertu de l'intérêt porté aux législations étrangères, comme cela avait été le cas en 1904 et en 1954. Effectivement, à toutes ces occasions, les juristes français en général et les comparatistes, en particulier, ont voulu réexaminer l'influence exercée par le Code $\mathrm{Na}$ poléon dans les autres pays. On peut même avoir eu l'impression qu'en 2004 l'Amérique Latine venait d'être redécouverte dans le domaine du droit comparé, comme elle l'avait été cinq siècles plus tôt du pont de vue historique et géographique.

Ainsi, dans le livre du bicentenaire, l'excellent rapport du Professeur Blanc-Jouvan au sujet de «L'influence du Code civil sur les codifications étrangères récentes » reconnaît l'existence de la pluralité des modèles et conclut que plusieurs systèmes sont en concurrence, chaque législation devant dégager, en fin de compte, la solution qui lui paraît la plus adéquate ${ }^{32}$. Dans ce rapport, les droits des pays en développement sont examinés à plusieurs reprises révélant un intérêt constant et une recherche et connaissance approfondies de chacune des législations citées. Dans ce même volume, le destin du Code Civil dans les pays d'Afrique et d'Amérique Latine a mérité plusieurs rapports représentant plus d'une centaine de pages ${ }^{33}$. Dans le même sens, l'étude du Professeur Claude Witz sur «L'influence des codifications nouvelles sur le Code Civil de demain » a examiné les législations récentes non seulement des pays industrialisés comme la Hollande et le Canada, mais aussi le nouveau Code brésilien ${ }^{34}$. De même il y a un rapport sur l'Amérique Latine dans l'ouvrage organisé par l'Université Panthéon-Assas Paris II intitulé « Le Code civil. Un passé, un présent et un avenir $»^{35}$.

Ces considérations nous prouvent qu'un important progrès se réalise dans la mesure où certains droits des pays en développement - en particulier ceux de l'Amérique Latine - ne sont plus exclus des préoccupations des comparatistes. Nous examinerons ensuite le

\footnotetext{
31 ibidem, ibidem, p. 892.

32 Xavier Blanc-Jouvan, In: Le Code Civil (1804-2004) Livre du bicentenaire. Paris: Dalloz-Litec, 2004. p. 483.

33 Le Code Civil (1804-2004) déjà cité, p. 515 et suivantes.

34 Ibidem, p. 651 et suivantes.

35 Le Code civil, un Passé, un Présent, un Avenir, Paris, co-publication de l'Université Panthéon-Assas, Paris II et de Dalloz, 2004.
}

rôle que certains de ces droits ont dans ce qu'on a fini par appeler le « bi-jurisdisme $»{ }^{36}$

En ce qui concerne les familles de droit, il n'y a aucun doute que le principe de l'exclusivité, selon lequel chaque droit appartenait à une seule famille ne s'applique plus nécessairement dans tous les cas. Comme cela a été bien remarqué par Jacqueline Flauss-Diem, il y a une certaine analogie entre l'évolution de la famille en droit civil et celle des familles en droit comparé de façon qu'à côté du modèle unique de jadis « il faut constater la présence des familles dites éclatées, recomposées, monoparentales, voire homoparentales ». Et l'auteur conclut que:

En définitive, la découverte du visage des enfants issus de ces unions constitue l'énigme à résoudre pour les comparatistes à l'aube de ce nouveau siècle. ${ }^{37}$

D'autre part, il n'y a aucun doute que les classifications proposées dans ce domaine sont basées sur la connaissance de la société qu'un droit déterminé gouverne et sur les techniques juridiques employées. Or, comme il est possible qu'une culture locale ou nationale subisse des influences les plus variées, il est évident qu'au pluriculturalisme doit correspondre un certain pluralisme des sources du droit et, au fond, un pluralisme juridique.

Finalement, le droit évoluant chaque fois plus rapidement, en passant des progressions arithmétiques aux progressions géométriques, il faut examiner les familles juridiques sous une perspective dynamique, comme l'affirme Ugo Mattei:

[...] 'les systèmes juridiques ne sont jamais. Ils sont toujours en devenir' et ce devenir est déterminé par le poids variable des modèles à l'intérieur des systèmes juridiques. ${ }^{38}$

Il y a, ainsi, dans chaque droit la possibilité de la présence ou de la coexistence de différents modèles, dont l'influence est variable, selon les époques et les domaines d'application, de telle façon que l'on peut même penser à une compétition entre les divers modèles et à une pondération variable de leur influence.

Relativisme, pluralisme et évolution permanente sont donc les caractéristiques que l'on retrouve dans les familles juridiques à partir du XXe siècle.

En appliquant ce critérium et en admettant les droits

\footnotetext{
36 Blanc-Jouvan, note bibliographique déjà citée, loc. cit., p. 889.

37 Jacqueline Flauss-Diem, op. cit., p. 203.

38 apud, Jacqueline Flauss-Diem, op. cit., p. 198.
} 
mixtes comme une troisième famille, il faudrait ajouter aux exemples cités par Vernon V. Palmer, dans son ouvrage, d'autres pays dont l'un représente une population supérieure à celle des sept États étudiés. C’est le Brésil, normalement inclut dans le système romano-germanique par les civilistes, qui ont raison de le faire, mais dont le droit public et, en particulier, le droit constitutionnel a été inspiré par les États-Unis. Des situations analogues peuvent exister dans d'autres pays, exigeant donc un nouvel examen du nombre des législations qui peuvent être considérées comme appartenant à la troisième famille ou peut-être même simultanément à cette dernière et à celle du droit civil. De n'importe quelle façon, une certaine révision des notions traditionnelles qui existaient en cette matière parait se justifier. Elle pourrait introduire ou consacrer des sous-systèmes dans les familles ou même aller plus loin et rompre l'équation du bi-juridisme pour accepter une pluralité de familles, dépassant la distinction classique entre common law et droit civil, ou même introduisant un nouvel élément de classification des droits, en adoptant, par exemple, un critérium complémentaire de nature économique.

En ce qui concerne la famille romano-germanique, il nous parait qu'il serait opportun de penser à un sous-système de droit comprenant l'Amérique Latine, considérant tant ses caractéristiques culturelles, économiques, politiques et sociales, que le rapprochement qui pourra se réaliser entre les diverses législations de la région, en vertu du Mercosur.

En effet, il existe une originalité des droits de l'Amérique Latine qui a été l'objet des études de René David $^{39}$, dans la mesure où ses citoyens sont simultanément « américains et latins ».40

Quoique l'on ne puisse pas parler, en réalité, d'un droit latino-américain, il n'y a aucun doute qu'il faut re-

\footnotetext{
39 René David écrit à ce sujet: « Certains auteurs latino-américains, Clovis Bevilaqua (Lições de direito comparado, 1897), et principalement Enrique Martinez Paz (Introducción al estudio del derecho comparado, 1934) ont revendiqué une place spéciale pour les droits de l'Amérique latine, parmis les différents systèmes de droit contemporains. Un point de vue plus nuancé a été soutenu par moi-même dans mon Traité élémentaire de droit civil comparé (1950): il m’est apparu que les droits latino-américains, tout en appartenant par leur structure et leurs traits généraux au système du droit continental, méritaient d'être classés, au sein de ce système, dans une catégorie à part, en raison de certains traits particuliers qui les distinguaient des autres droits du système ». (Le Droit Comparé Droits d'Hier, Droits de Demain, op. cit., p. 165).

40 René David, Traité Élémentaire de Droit Civil Comparé. Paris: LGDJ, 1950. p. 259
}

connaître l'existence d'un groupe de droit, ayant certains dénominateurs communs, qui sont en vigueur en Amérique Latine. Cette situation a été décrite par René David dans son Traité ${ }^{41}$ mais, depuis lors, peu d'études ont reconnu cette position que devrait avoir le droit de l'Amérique Latine. En réalité, la question a été posée ${ }^{42}$, mais est restée sans réponse dans la majorité des travaux des comparatistes.

\section{Un critérium économique pour une classification complémentaire en Droit Comparé}

Ainsi, au lieu de réunir sous un titre unique tous les droits qui ont subi une double influence, en les étiquetant comme appartenant à une troisième famille, il serait peut-être plus logique et rationnel de créer des sous-systèmes dans les cas où une des influences prévaut, exerçant une espèce d'hégémonie. Dans d'autres situations, de nouvelles classifications devraient être proposées et discutées.

La dénomination de troisième famille peut être interprétée comme une espèce d'exclusion, puisqu'il s'agit d'une catégorie où l'on réunit tous ceux qui n'appartiennent pas exclusivement à une des deux grandes familles. Effectivement, rien d'autre n'unit les uns aux autres l'Écosse, l'Afrique du Sud, Porto Rico et Israël.

Certains auteurs ont voulu opposer le monde occi-

\footnotetext{
41 René David (Traité op. cit., p. 267) développe son analyse dans les termes suivants: «Les conditions de ce milieu sont telles qu'elles imposent, à certains égards, de considérer les choses et d'organiser la vie sociale autrement en Amérique qu'en Europe. De même que la civilisation latine s'est transformée dans le temps, de même elle se transforme dans l'espace, et elle revêt un aspect différent des deux côtés de l'Atlantique. Les droits des pays latins d'Amérique, faits pour s'appliquer dans un milieu différent du milieu américain, se distinguent des droits de l'Europe continentale et constituent, au sein du groupe du droit français, une catégorie originale, dont les traits spécifiques apparaissent de plus en plus clairement au fur et à mesure que le divorce s'atténue entre des textes jadis théoriques et les réalités de la vie. »

42 René David et Camille Jauffret-Spinosi. (Les grands systèmes de droit contemporains, Paris, Dalloz, 1992, p. 57) écrivent que: « La question qui se pose est seulement de savoir dans quelle mesure les conditions propres à l'Amérique, différentes de celles du milieu européen, ont conduit ou peuvent conduire à reconnaître ou conférer une certaine originalité aux droits de l'Amérique par rapport aux droits européens de la famille romano-germanique ».
} 
dental au reste du monde, mais ce critérium n'est pas non plus correct, l'adhésion aux valeurs de l'Occident n'étant pas toujours du même degré et pouvant correspondre à des techniques juridiques très différentes.

Finalement, on peut imaginer une nouvelle classification basée sur les niveaux économiques de développement des pays, qui ont des répercussions sur leur droit.

Dans les dernières années, il y a eu un rapprochement important entre le droit et l'économie, non seulement aux États-Unis ${ }^{43}$, mais aussi en Europe et au Brésil, dans la mesure où la doctrine a reconnu l'existence successivement du droit économique et du droit public de l'économie ${ }^{44}$. D'autre part, de la même façon que s'est affirmée l'existence d'une économie du développement, il faut reconnaitre que, depuis quelques années, plusieurs travaux se concentrent sur le droit du développement tant dans le domaine international qu'interne ${ }^{45}$.

La comparaison entre les droits des pays industrialisés et ceux du tiers monde ou entre les législations des États en voie de développement, les uns avec les autres, peuvent présenter un intérêt en même temps académique et pratique. La mondialisation a créé une nouvelle donne et une interdépendance croissante entre les diverses régions du monde, de façon qu'il est important de connaitre et approfondir les instruments juridiques qui permettent d'assurer la stabilité des marchés et le développement soutenu et durable tant des pays du tiers monde que de ceux considérés comme étant en voie de développement.

43 Richard A. Posner, Frontiers of Legal Theory, Cambridge, Harvard University Press, 2001, p. 31 et suivantes, et du même auteur, Overcoming Law. Cambridge: Harvard University Press, 1995. p. 406 et suivantes.

44 Bruno Deffains (sous la direction de), L'Analyse Économique du Droit dans les Pays de Droit Civil, Préface de Guy Canivet, Paris, Éditions Cujas, 2002; perspectives du Droit Économique - Dialogues avec Michel Jeantin, Paris, Dalloz, 1999; François Servoin, Droit Administratif de l'Économie, 2eme édition, Grenoble, Presses Universitaires de Grenoble, 2001; D. Linotte et A. Mestre, Services Publics et Droit Public de l'Économie. Paris: Litec, 1982.

45 Robert Charvin, L'Investissement International et le Droit au Développement, Paris: L'Harmattan, 2002; Cláudia Perrone-Moisés, Direito ao Desenvolvimento e Investimentos Estrangeiros, São Paulo: Ed. Oliveira Mendes, 1998; Ana Paula Texeira Delgado, O Direito ao Desenvolvimento na Perspectiva da Globalização: Paradoxes e Desafios. Rio de Janeiro: Renovar, 2001; Arnoldo Wald, «Il Diritto dell'Economia e il Diritto dello Svilupo in Brasile ", In: Diritto dei nuovi mondi. Atti del convegno promosso dall'Instituto di Diritto Privato delle Facoltá di Giurisprudenza. 5-7 nov. 1992, Genova: CEDAM, 1994, p. 176-191; Celso D. de Albuquerque Mello, Direito Internacional Econômico, Rio de Janeiro: Renovar, 1993.
L'étude des systèmes ou "systémalogie juridique » selon la dénomination que lui donne le Professeur RoDOLFO SACCO devient ainsi « un instrument de connaissance du droit interne et de vérification des hypothèses concernant l'analyse sociologique du droit ». C'est également « l'élément médiateur entre science juridique et histoire (et) entre science juridique et théorie générale du droit $»^{46}$.

Dans un monde imprévisible, dans lequel les prophéties ne se réalisent souvent pas, une grande banque américaine vient de construire un scénario, pour les années 2050, dans lequel quatre des pays qui sont encore aujourd'hui considérés comme ayant une économie en développement deviendraient les plus importants du point de vue mondial : la Chine, l'Inde, la Russie et le Brésil ${ }^{47}$. L'éventuelle ascension de ces pays connus sous la dénomination de BRIC (Brésil, Russie, Inde, Chine) présuppose toutefois de grandes transformations sur le plan juridique et sur celui des institutions pour chacun d'entre eux.

Ainsi, il serait possible d'imaginer un sous-système de la famille romano-germanique, comprenant les pays en voie de développement, entre lesquels ceux de l'Amérique Latine.

D'un autre côté, une comparaison pourrait être faite entre les pays en phase de grandes transformations économiques et sociales, complétant ainsi une classification purement juridique, culturelle et technique, par une autre qui pourrait être inspirée par les niveaux atteints par le développement juridique et institutionnel et la vitesse des transformations des structures de certains États. Il serait ainsi possible de vérifier, sur le plan juridique, ce qu'il y a de commun entre des pays comme le Brésil, la Chine, l'Inde et la Russie. Peut-être que les analogies ne seraient pas moindres que celles que VERNON PALMER signale entre les sept États qui, selon sa conception, constitueraient la troisième famille juridique.

L'examen des familles juridiques et de leur classification au début du troisième millénaire justifie une révision des conceptions qui dominaient le XXème siècle. Il est donc important de poser des questions à ce sujet et

\footnotetext{
46 Rodolfo Sacco, La Comparaison Juridique au Service de la Connaissance du Droit. Paris: Economica, 1991. pp. 108-111.

47 Études de Goldman Sachs (Global Economics Papel, $n^{\circ}$ 99) intitulé « Dreaming with BRICs: The Path to 2050 ». La revue Marianne du 19 au 25.01.2004 fait reférence à ce travail dans un article intitulé "Ces géants qui domineront le monde de demain ", par Thomas Vallières (p. 52).
} 
de proposer de nouvelles formules qui soient adaptées à un monde juridique agrandi et plus complexe que celui que nous connaissions jadis et qui ne se limite plus à l'Europe et aux États Unis et où l'on assiste sinon à un choc, ou moins à une complémentarité des cultures et des économies.

En conclusion, à côté de la comparaison verticale qui correspondait au monde où le droit était considéré comme ayant une structure pyramidale, il nous faut admettre une comparaison horizontale « infiniment plus fructueuse » à laquelle fait allusion le Profeseur BLANC-JOUVAN ${ }^{48}$.

Dans une époque dominée par le droit à géométrie variable, la doctrine est déjà arrivée à considérer que la figure géométrique la plus adéquate pour nous donner une vision claire de l'utilisation des modèles serait:

\footnotetext{
« celle d'un triangle isocèle dont les trois angles sont constitués chacun par un modèle, les différents systèmes juridiques se plaçant sur les cotés avec une proximité plus ou moins grande de l'un des angles 'modélisés'. » ${ }^{49}$
}

Peut-être, serait-il également possible de faire des comparaisons en diagonale, en rapprochant des systèmes de droit nationaux inclus parmi des familles juridiques différentes mais dont les niveaux de développement des institutions juridiques seraient d'un même degré ou évolueraient avec la même vitesse.

Tout cela nous ramène à la conclusion que le droit comparé retrouve un nouveau souffle et une importance croissante, comme instrument de compréhension et tolérance entre les pays, comme pont entre les cultures différentes et comme catalyseur de la coopération et de la solidarité internationale.

Il nous faut donc reconnaitre avec le Professeur Blanc-Jouvan que:

Si le pluralisme est aujourd'hui à l'ordre du jour, c'est indiscutablement qu'il représente une solution d'avenir : et les questions qu'il pose sont appelées à se multiplier.50

\section{Le Droit Comparé dans la formation et dans la pratique des juristes au Brésil}

$48 \quad$ Xavier Blanc-Jouvan, note citée, p. 891.

49 Ugo Mattei, apud, Jacqueline Flauss-Diem, op. cit., p. 201.

50 Xavier Blanc-Jouvan, note bibliographique citée, p. 894.
L'appréhension du droit comparé sous un angle autre que celui de la recherche juridique pure présente une certaine difficulté dans la mesure où il n'a jamais bénéficié de la reconnaissance unanime de sa légitimité dans l'enseignement ou dans la pratique. La situation ambiguë de cette matière résulte de divers facteurs, le plus significatif étant que les facultés de droit enseignent le droit positif interne, le droit international ne visant en réalité qu'à accorder une application extra nationale de règles issues d'un ordre juridique interne. De même, en ce qui concerne la pratique, le juge a pour fonction d'appliquer le droit interne, ce qui se répercutera nécessairement sur les sources utilisées par les avocats, en donnant une plus grande importance au droit national, et se limitant, au maximum, à citer quelques règles de droit étranger, comme illustration ou élément d'interprétation des lois locales.

Ainsi, le droit comparé apparaissait classiquement comme une matière «exotique» sans grande utilité aux yeux de nombreux professionnels de l'enseignement ou de la pratique. Néanmoins, la formation de l'Etat brésilien, et donc de son ordre juridique, a subi les multiples influences des populations qui ont participé à la formation de la Nation brésilienne, généralement présentée comme un exemple de métissage et d'assimilation. En ce sens, personne ne peut nier que le droit national brésilien ne serait ce qu'il est aujourd'hui sans avoir pu croître sur le fertile terreau de la culture juridique occidentale.

De cet incontestable état de fait, on doit déduire la différence structurelle entre les droits des nations plus anciennes et l'ordre juridique brésilien, le droit étranger ayant, implicitement ou explicitement, bénéficié d'une plus forte attention de la part des juristes brésiliens que de leurs homologues du Premier Monde. Aujourd'hui, le Brésil n'étant plus une terre vierge à conquérir, cet aspect originel du droit brésilien devrait naturellement tendre à s'estomper, l'expérience acquise par le juriste brésilien l'autorisant à s'affranchir, en partie, de ses modèles importés.

Nous tenterons ainsi de présenter les traits les plus saillants de l'importance actuelle du droit comparé au sein de la formation et de la pratique du droit, tant en fonction des acquis historiques que des perspectives de l'environnement du juriste.

Lors d'une conférence prononcée à la Faculté Nationale de Droit, à l'occasion de l'inauguration de l'année 
académique de 1955, le Professeur San Tiago Dantas a tenté de définir l'objet de la formation du juriste, donc également la fonction de l'enseignement juridique universitaire, en affirmant que «l'université a pour rôle l'élaboration des nouveaux instruments de la culture que réclame la vie sociale ", sans préjudice de l'enseignement professionnel. ${ }^{51}$

La place réservée au droit comparé, lors de la formation de l'étudiant en droit, sera appréciée simultanément à la présentation des divers aspects du cursus universitaire juridique, dont il conviendra tout d'abord de dessiner rapidement la structure institutionnelle, pour ensuite décrire brièvement l'organisation et la durée des enseignements, afin de terminer par les modes d'accès à la magistrature et à la profession d'avocat.

Il convient d'abord de mentionner l'importance relativement forte de l'enseignement supérieur privé au Brésil comparé à celui de certains pays européens.

L'enseignement public, même si le Brésil dispose d'une organisation fédérale, la distribution des compétences en matière d'enseignement supérieur n'est pas exclusive, ce qui amène à une certaine atomisation des structures. Il existe donc un réseau d'universités gérées par l'Etat, sous la responsabilité de l'Union Fédérale ou des Etats-membres. Parmi les plus anciennes, il faut citer celles de Recife et de São Paulo, mais se détachent également d'autres écoles, parmi lesquelles celles de Rio de Janeiro, Porto Alegre, Belo Horizonte, Brasilia et Salvador. En outre, on ne peut manquer de mentionner le réseau des Universités catholiques, qui possèdent, au Brésil, un prestige et une reconnaissance internationale égale à ceux des universités publiques, la qualité des enseignements et des travaux de recherche qu'elles produisent étant incontestable. Enfin, il y a également quelques centaines de facultés privées dont les niveaux culturels et pédagogiques sont très inégaux, malgré le contrôle exercé par l'Etat.

Le contenu de l'enseignement juridique est déterminé au niveau national par le Ministère de l'Education, auquel doivent se conformer toutes les entités publiques ou privées délivrant ces formations.

L'étudiant juriste commence théoriquement son cursus universitaire aux alentours de 18 ou 19 ans, après

\footnotetext{
51 San Tiago DANTAS, «A Educação Jurídica e a Crise Brasileira: aula inaugural dos cursos da Faculdade Nacional de Direito, em 1955 », In: Palavras de um Professor. Rio de Janeiro: Forense, 1975. p.79.
}

l'obtention du vestibular, qui vise à permettre l'entrée de l'étudiant à l'université. Ensuite, le premier diplôme de droit, le bacharelado (licence), ne s'obtient qu'au bout de quatre ou cinq années d'études, dites de graduação, au cours desquelles l'étudiant devra obtenir son approbation dans un nombre minimum de matières.

En licence, l'enseignement du droit comparé n'existe pas en tant que tel, même s'il a déjà existé un cours de « législation comparée » au début du siècle. Ce n'est donc qu'en maitrise ou doctorat que les juristes étudieront de manière plus approfondie le droit comparé. Même à ce niveau, il n'y pas, en général, de cours concernant la méthode de droit comparé et l'étude des grands systèmes juridiques. Les professeurs d'une matière déterminée l'examinent donc sous l'angle du droit comparé. Le civiliste enseigne le droit civil comparé ou le droit de la famille comparé. De même, le publiciste traite une institution ou une technique en l'analysant dans les diverses législations, comme ce serait le cas, par exemple, du contrôle de la constitutionnalité des lois.

Cependant, dès les cours de licence l'étudiant sera confronté, en réalité, dans toutes les matières, à une présentation plus ou moins succincte des régimes juridiques étrangers. A titre d'exemple, l'apprentissage du droit civil ne peut se passer de traiter des concepts de base venant du droit étranger, comme bien évidemment le droit romain, mais aussi le Code Napoléon ou le BGB allemand, dans des proportions bien plus importantes que cela se fait normalement en Europe et même aux Etats-Unis. Auparavant, l'étudiant, à ce stade, bénéficiait d'une formation générale humaniste, orientée vers une optique de rhétorique juridique, pour lui permettre d'assimiler tant des concepts de pur droit que de philosophie, d'histoire ou de sociologie et de politique.

En effet, pendant longtemps, les facultés de droit avaient une fonction plus culturelle et moins professionnelle. A cette époque, tant les professeurs, dans leurs cours, que les manuels de droit destinés aux étudiants, ne donnaient pas une grande importance à la jurisprudence et certains traités juridiques la dédaignaient même. Ce n'est qu'à partir des années 1960 que le divorce qui existait entre la doctrine et la jurisprudence a été dépassé et que, d'autre part, les professeurs et les juristes en général ont commencé à attribuer une certaine importance, dans l'enseignement du droit national, aux données sociales et économiques. Cela a été un premier pas pour pouvoir aborder d'une façon rationnelle et scientifique 
le droit comparé, en ne limitant plus l'étude du droit étranger à la simple citation des articles de loi.

Aujourd'hui, la méthode d'enseignement tend à équilibrer la formation entre les cours magistraux et les travaux pratiques, les dernières réformes ayant été motivées par le caractère trop théorique de l'enseignement en égard aux besoins des professionnels. Désormais, l'étudiant se spécialise à partir de la quatrième année de graduação, pouvant notamment choisir entre une dominante de droit public ou de droit privé. Dans certaines facultés, à coté des matières obligatoires, l'étudiant peut choisir certains cours facultatifs.

En ce qui concerne les professions juridiques, le titre de bacharel (licencié) permet de se présenter aux concours de la magistrature et aux examens qui permettent l'inscription au Barreau (Ordre des Avocats du Brésil). La connaissance des législations étrangères ne constitue pas une des conditions de la réussite de ces concours, mais il faut toutefois noter que, lors des épreuves, les étudiants se voient évaluer d'une façon assez sommaire sur leur culture juridique et doivent donc prouver leur maitrise des principaux concepts juridiques de notre droit, qui s'inspire de la civilisation occidentale.

Comme nous le voyons, le droit comparé en est à ses débuts au Brésil, malgré l'effort fait ces dernières années. Nous sommes encore assez loin de l'idéal auquel fait allusion le Professeur Sacco quand il propose que le navire gagne son port, après les nouvelles batailles que doivent mener les juristes, qu'ils soient italiens ou brésiliens, et en vertu desquelles:

Le professeur qui enseigne le droit territorial se rend compte de l'avantage qu'assure à l'étudiant pour la compréhension de son droit national - la connaissance du droit comparé. De là, il n'y a qu'un seul pas à faire pour trouver deux vérités. Toute matière juridique devra être enseignée deux fois: sous l'angle comparatif et sous l'angle national. Ensuite l'enseignement du droit comparé devrait précéder et non suivre l'étude du droit national. ${ }^{52}$

\section{Le Droit Comparé dans la pratique du Droit au Brésil}

Le praticien, le juge ou l'avocat, ne peut plus ignorer le droit étranger. Les nécessités de la pratique

\footnotetext{
52 Rodolfo SACCO, « La formation au droit comparé, l'expérience
} italienne ». Revue internationale de droit comparé, 1996. p. 274. imposent de se soucier des droits étrangers. Ce sont, en effet, les besoins de la pratique qui obligent le juriste à s'informer, à s'efforcer de comprendre d'autres règles ou d'autres cultures. ${ }^{53}$

Comme il l'a été précisé, le droit comparé a toujours eu une place remarquable dans la vie des juristes brésiliens. Les lois portugaises ayant été applicables au Brésil après sa déclaration d'indépendance, la connaissance du droit portugais était donc un préalable à l'institution et à la compréhension de l'ordre juridique auquel allait se soumettre le nouvel Etat. Lacunes législatives au temps de la suprématie de la loi véhiculée par la doctrine de l'exégèse, absence d'une littérature juridique brésilienne et besoins de réglementation ont contribué à faire de la recherche comparative une condition sine qua non de l'exercice des professions juridiques au Brésil. Ainsi, à cette époque, le recours aux conceptions juridiques tirées de la doctrine étrangère n'était pas un fruit de l'érudition de quelques juristes plus curieux, mais, au contraire, une vraie nécessité pour l'exercice de la profession.

Plus tard, avec l'institution d'une législation brésilienne, l'élargissement de la notion de source de droit et la création des Facultés de Droit, une culture juridique proprement brésilienne a commencé à se développer. Toutefois, cela n'a pas suffit à rayer de l'esprit des juristes brésiliens l'habitude, devenue un réflexe, de la recherche comparative. Aujourd'hui encore, le juriste étranger qui viendrait à pratiquer son activité au Brésil serait frappé par la richesse des références aux textes de loi étrangers, à la jurisprudence ou à la doctrine d'autres pays que les praticiens du droit brésilien utilisent quotidiennement. Un ouvrage, un article, une consultation ou tout autre type de travail juridique ne sont pas considérés dignes de ce nom sans une référence aux solutions des autres ordres juridiques. Ayant déjà examiné l'influence du droit étranger sur le législateur, nous présenterons succinctement le recours aux modèles étrangers dans la pratique des magistrats, puis dans l'exercice de la profession d'avocat.

\subsection{Le droit comparé et la jurisprudence brésilienne}

Il y a 70 ans que René David, en décrivant la prati-

\footnotetext{
53 Georges FLÉCHEUX, «La situation en France, le point de vue des professions juridiques », Revue internationale de droit comparé, 1996. p.320.
} 
que juridique au Brésil et plus spécifiquement celle des juges, a conclu:

Avec la sécheresse des jugements français, et avec l'ignorance qu'ils manifestent extérieurement de la doctrine, le contraste est frappant si l'on lit les arrêts des juges brésiliens. Au Brésil, la doctrine, tant étrangère que nationale, paraît jouer un rôle de premier plan: auteurs français, italiens, portugais, espagnols, argentins sont constamment cités, commentés par les juges dans leurs arrêts. Quiconque étudie une question de droit brésilien doit en tenir compte, et ne pas prétendre résoudre cette question en considérant les seules lois, décisions judiciaires, opinions des auteurs du Brésil. Chaque droit doit être étudié selon les méthodes propres qu'il comporte, faute de quoi on ne saurait arriver à vraiment le connaitre.» ${ }^{54}$

A titre d'exemple de l'influence du droit comparé sur les tribunaux, on peut rappeler que, pour justifier la révision des contrats et même l'indexation des dettes, en période d'hyperinflation, la Cour Suprême a considéré que la théorie de l'imprévision et la révision par le pouvoir judiciaire constituaient de vrais principes généraux applicables par le juge brésilien. Le rapporteur a, à l'occasion, invoqué l'interprétation donnée par les tribunaux allemands de l'article 242 du BGB qui fait allusion à la confiance et à la bonne foi qui doivent inspirer l'exécution des contrats (treu und glauben).

De même, un arrêt de la Cour Suprême (Supremo Tribunal Federal) du 5 mai 1978, qui, en appréciant les effets éventuels d'un acte administratif irrégulier, annulé en vertu du changement de l'interprétation donnée à un texte par les autorités, a discuté de l'application, en droit brésilien, de la décision du Conseil d'Etat français dans l'affaire Dame Cachet. A cet effet, le Ministre Leitão de Abreu a, dans son vote, considéré que le droit brésilien n'admettait pas cette orientation, mais il a préalablement examiné les commentaires de Maurice Hauriou et de Marcel Waline et la position de Léon Duguit, comme s'il s'agissait d'une affaire de droit français ${ }^{55}$.

D'autre part, si le juge brésilien n'applique pas, en général, une loi d'un autre pays, il n'hésite pas à faire référence aux solutions étrangères. Il y a même des juges qui utilisent des textes étrangers et n'hésitent pas à les citer, à titre d'information, au cours des jugements et

\footnotetext{
54 Réné DAVID, Traité élémentaire de Droit compare. Paris: Economica, 1982. p. 243.

55 Recurso Extraordinário n. 88.214-SP, publié dans la Revista Trimestrial de Jurisprudência do Supremo Tribunal Federal, v.88, 1979. p.278.
}

dans leurs arrêts ${ }^{56}$.

L'importance de la recherche comparative devient encore plus importante lorsque le juge brésilien est obligé de soumettre la résolution d'une situation juridique déterminée au droit indiqué par sa règle de conflit. Aux yeux du juge brésilien, la loi étrangère n'est pas une question de fait, mais de droit, ce qui implique l'application de règles juridiques étrangères par le juge brésilien, même si ces règles n'ont pas été invoquées par les parties. En pratique, cela n'arrive que très rarement, en vertu de clauses contractuelles, lors des actions de nullité ou d'annulation des arbitrages internationaux ou enfin à l'occasion de l'examen, par le juge, de la validité des effets d'actes juridiques pratiqués à l'étranger ${ }^{57}$.

Autant la Cour Suprême que le Supérieur Tribunal de Justice ont eu l'occasion de discuter la jurisprudence et la doctrine françaises en ce qui concerne la définition des droits acquis, malgré le fait que cette matière ait mérité une protection constitutionnelle au Brésil, tandis qu'en France il s'agit d'une question réglée par la loi. ${ }^{58}$

De même, le principe d'égalité qui doit être reconnu par l'acte administratif a été analysé par le Supérieur Tribunal Justice en se fondant sur les enseignements de Gaston Jèze. ${ }^{59}$

En d'autre matière, comme, par exemple, la responsabilité civile en général et les clauses d'exonération du transporteur, il y a également de nombreuses références de la jurisprudence au droit français et à la doctrine étrangère, comme l'a signalé le Conseiller Cláudio Santos. ${ }^{60}$

56 Il est intéressant de noter, par exemple, qu'au sein de la Cour Suprême Fédérale brésilienne, il est récemment devenu fréquent de faire référence au contenu des jugements d'autres cours constitutionnelles - et même au contenu des arrêts des cours supranationales telles que la Cour Interaméricaine des Droits de l'Homme - qui ont été adoptés comme paramètre interprétatif por l'application des règles de droit brésilien.

57 La fréquence faible de l'application des règles de droit étranger par le juge national au Brésil est historiquement associée à la tendance territorialiste des règles de Droit International Privé au pays, dont les critères de rattachement favorisent l'application du droit brésilien. Au même temps, elle est aussi associée au profil traditionnellement opaque, omis, peu concluante de la législation brésilienne à propos de la liberté de choix des parties quant à la loi applicable à leur rapport juridique.

58 Recurso Especial no 506-RJ, publié dans la Revista do Superior Tribunal de Justica, n. 6, 1990, p. 360.

59 Mandado de Segurança n. ${ }^{\circ}$ 80, jugé par le STJ.

60 Claudio SANTOS, «L'influence du droit français sur la jurisprudence du Supérieur Tribunal de Justice », Rapport des Journées franco-italiennes de l'Association Henri Capitant, Travaux de l'Association 


\subsection{Le droit comparé et la pratique des avocats}

Le juriste européen débarquant au Brésil ne tardera pas à s'habituer au système législatif, cela aussi bien sous les aspects processuels que matériels du droit, Tullio Ascarelli a également remarqué que les particularités du droit brésilien augmentent quand le voyageur juridique se tourne vers l'observation de la pratique. D'après le Professeur de Bologne, le visiteur va vite s'apercevoir que les doctrines françaises et italiennes sont largement utilisées dans la pratique. Il pourra également observer que ces doctrines sont même plus souvent invoquées que la doctrine allemande, et cela en dépit de l'influence germanique dans l'élaboration du Code Civil de 1916, ce qui sans doute peut s'expliquer par la facilité dans l'apprentissage des langues latines par rapport aux langues germaniques ${ }^{61}$.

Il faut toutefois prendre en considération que ces analyses descriptives réalisées par René David et Tullio Ascarelli se limitent à ce qu'ils ont pu constater pendant les années 1950. Il faut donc donner suite à cette analyse en tenant compte de la récente évolution de la profession d'avocat. Cela étant, à deux moments distincts de l'histoire récente les juristes brésiliens ont été plus sensibles à l'introduction de nouvelles habitudes d'origine étrangère dans leur quotidien.

Tout d'abord, en vertu et à la suite de la Deuxième Guerre Mondiale, les acteurs du commerce international se sont retrouvés en face de l'incontestable hégémonie commerciale nord-américaine qui a perduré, en Amérique Latine, beaucoup plus longtemps et d'une façon beaucoup plus marquante qu'en Europe. Or, ce changement des pratiques commerciales et, plus généralement, de l'économie brésilienne, n’ont pas été sans conséquence sur la réforme du droit et sur l'exercice des professions juridiques au Brésil, dans une reproduction du modèle de relation entre l'infrastructure et la supers-

Henri Capitant, Tome XLIV. Paris: Litec, 1994. p. 137-139.

${ }_{61}$ Tullio ASCARELLI; Studi di Diritto Comparato, Milano, Giuffrè, 1952, p. 83. Dans l'original: «Ma la sensazione della peculiarità del diritto brasiliano verrà aumentando quando il viaggiatore giuridico si rivolgerà alla pratica, a quella contrattuale, a quella processuale e a quella che vorrei dire dottrinaria, e cioè al modo col quale i problemi, ancor prima che risolti, vengono impostati nella dottrina. Noterà immediatamente la larga utilizzazione nella pratica della dottrina francese e italiana, e potrà anche osservare come siano appunto que ste dottrine quelle che, anche (ma non solamente) per ragioni linguistiche, sono le più invocate, nonostante gli elementi germanici del codice civile. » tructure d'une société.

Ensuite, depuis une trentaine d'année, avec la mondialisation, l'ouverture de l'économie brésilienne et le mouvement d'intégration du Cône Sud de l'Amérique Latine, la présence et les investissements étrangers se sont multipliés, ainsi que les contacts tant avec les Etats-Unis qu'avec l'Europe.

$\mathrm{Au}$ cours de ces deux périodes, nous avons pu observer, tout d'abord, l'occurrence d'une certaine américanisation du droit privé brésilien. Cela a entrainé l'introduction de certaines habitudes nord-américaines dans la vie et dans la pratique des juristes brésiliens. La réception de ces habitudes s'est premièrement manifestée par l'adoption d'un vocabulaire rempli d'anglicismes. La convivialité entre les opérateurs du droit au Brésil et leurs collègues ou clients de formation nord-américaine rend plus naturelle l'insertion de paroles telles que due diligence dans les dialogues en portugais. Par ailleurs, depuis déjà quelques temps, le jeune avocat cherchant à travailler dans un cabinet moderne ne trouvera de place que s'il n'a pas, au moins, une bonne connaissance de la langue anglaise.

La notion de ce que l'on considère au Brésil comme étant un cabinet d'avocats moderne passe nécessairement par l'adoption d'un schéma d'organisation largement inspiré du modèle des law firms nord-américaines. Il faut également mentionner, en tant que facteur d'acculturation juridique, l'installation au Brésil de quelques-uns des plus grands cabinets nord-américains, qui exercent la profession à coté des cabinets traditionnels, ce qui a entraîné une augmentation de la qualité des services offerts par les juristes brésiliens. De nos jours, il ne suffit plus de parler la langue des clients et des collègues étrangers pour garantir la quantité et de la qualité des services. Il devient impératif également de bien connaître les droits étrangers. Cela constitue un facteur de la plus grande importance puisqu'il permet aux praticiens du droit de pouvoir passer outre leurs différences culturelles, ce qui rend toute négociation plus facile.

Ces besoins du commerce international encouragent les cabinets brésiliens à envoyer leurs professionnels à l'étranger pour des stages et des périodes de formation et les ont même motivés à engager quelques avocats et stagiaires d'origine étrangère. Aujourd'hui, comme nous pouvons le constater par l'examen de certains annuaires internationaux, un bon nombre des juristes au ser- 
vice des cabinets brésiliens ont une formation et une expérience internationale, qui peuvent être placées au niveau des cabinets d'Amérique du Nord ou d'Europe, et connaissent donc le droit étranger, devenant éventuellement, à certains moments, des comparatistes par nécessité dictée par leur clientèle.

Ensuite, cette évolution correspond également aux mutations du contexte national et régional du Brésil depuis la fin des années 1980. Ce n'est qu'après la chute des régimes militaires d'orientation nationaliste, jusqu'alors présents en Amérique Latine, qu'une plus grande libéralisation des rapports commerciaux entre le Brésil et ses voisins sud-américains a été possible. Encore incomplet, ce rapprochement a été relativement rapide, surtout quand on se souvient que, jusqu'au milieu des années 1980, la moitié des troupes de l'armée brésilienne se concentrait encore près de la frontière argentine, alors qu'aujourd'hui les ressortissants des deux pays n'ont plus besoin d'un passeport pour la traverser. Les négociations en vue d'approfondir l'intégration du Cône Sud de l'Amérique Latine au sein des institutions du Mercosul existantes ont déjà fait prendre conscience, à tous les acteurs de la région, de la nécessité d'harmoniser les législations, ce qui passe déjà par la connaissance des droits des partenaires.

De même, la reconnaissance mutuelle des diplômes délivrés par les institutions d'enseignement d'un pays-membre constitue un autre obstacle à la libre prestation de services, dont la résolution pourrait s'inspirer du droit communautaire.

En ce qui concerne la pratique des avocats étrangers au Brésil, qui n’ont pas revalidé leurs diplômes, certaines formules constructives ont été trouvées et d'autres tolérées, afin de trouver une solution adéquate. La présence de ces avocats étrangers au Brésil est également un catalyseur de la meilleure compréhension du droit étranger.

Finalement, pendant les trente dernières années, l'ouverture de l'économie brésilienne et les privatisations, ainsi que le déclin de l'activité de l'Etat comme opérateur financier, commercial, et industriel, ont exigé et permis de très importants investissements étrangers dans des secteurs aussi diversifiés que la banque, l'électricité, le pétrole, la construction des routes, l'assurance ou la réassurance. Parmi les grands investisseurs, on retrouve, après les Etats-Unis, la Chine, la
France, l'Allemagne, la Grande-Bretagne, l'Espagne, le Portugal, l'Italie et les Pays-Bas. Ce mouvement a eu un très grand impact sur l'étude des législations étrangères, tant en ce qui concerne le régime des investissements que sur celui applicable à la progressive libéralisation économique.

\section{Les activités de Droit Comparé au Brésil}

L'impératif est dans l'immédiat, de développer et surtout de coordonner les actions engagées en faveur du droit comparé. ${ }^{62}$

Nous avons déjà vu qu'il n'existe pas, pour le moment, de chair de droit comparé, d'études des grands systèmes juridiques ou de droit étranger dans les facultés, quoiqu'elles aient existées, à la fin du XIXe siècle et au début du XXe siècle, sous la dénomination de législation comparée. Nous n'avons pas de juristes qui se dédient exclusivement au droit comparé, cette branche du droit étant l'objet d'une seconde activité éventuelle, pour des professeurs, magistrats et avocats spécialisés en droit public ou privé.

Le Brésil dispose de plusieurs instituts, liés ou non à des facultés, ayant des activités se rapportant au droit comparé, mais ils n'ont que des structures réduites, et se limitent à la publication d'une revue ou de travaux divers et à l'organisation de séminaires ou de congrès ${ }^{63}$.

Après la Seconde Guerre Mondiale, un renouveau des études de droit comparé s'est fait sentir, sous l'influence de plusieurs juristes européens que l'exil avait amené au Brésil, comme Tullio Ascarelli et Enrico Tullio Liebman, tous deux professeurs à São Paulo pendant leur séjour dans notre pays à cause de la guerre.

Ainsi, en 1949, l'Institut de droit comparé de Rio de Janeiro a été créé, sous la Présidence du Professeur San Tiago Dantas, disposant de moyens importants et d'une grande bibliothèque, mais il n'a pas résisté plus que quelques années, le contexte de l'époque ne justifiant pas un

\footnotetext{
${ }^{62}$ Xavier BLANC-JouVAN, « La formation au droit comparé, le point de vue d'un universitaire ». Revue internationale de droit comparé, 1996 , p. 347.

63 Ainsi l'Institut de Droit Comparé brésilien a publié avec la Société de Législation Comparé le livre Le droit brésilien d'bier, d'aujourd'bui et de demain, Dir. par Arnoldo Wald et Camille Jauffret-Spinosi, Paris, Société de Législation Comparée, 2005.
} 
grand intérêt du public académique ni des professionnels pour cette matière.

Dès cette période, des groupes de juristes brésiliens, professeurs, avocats et magistrats, se sont réunis en vertu de leurs liens avec des associations internationales de droit comparé, comme c'est le cas des groupes brésiliens tant de la Société de législation comparée que de l'Association Henri Capitant. Le premier, avec une cinquantaine de membres, est le plus important entre ceux des pays étrangers, pouvant se comparer au groupe italien, et la Société a réalisé plusieurs congrès au Brésil, ses membres ayant, à plusieurs reprises, eu l'occasion de publier leurs études dans la Revue internationale de droit comparé.

Le Comité brésilien de droit comparé créé dans les années 1950, sous la présidence du Professeur Haroldo Valladão, a maintenu ses activités pendant une trentaine d'année, avec des réunions mensuelles et la présentation de travaux lors des congrès internationaux.

Deux instituts sont actuellement plus liés au droit comparé. Il s'agit de la Bibliothèque Tullio Ascarelli de la Faculté de Droit de São Paulo et l'Institut de Droit Comparé Luso-Brésilien, cette première institution réalisant d'importants travaux de droit commercial en général, mais également de droit comparé dans ce domaine, avec une revue qui parait chaque trimestre et qui a dépassé la centaine de numéros. A Rio de Janeiro, l'Institut de droit comparé luso-brésilien a été créé en 1982, et s'occupe plus directement de la comparaison entre les droits brésiliens et portugais, organisant des congrès, au Portugal et au Brésil, et publiant sa revue annuelle.

Les questions de droit comparé sont également soulevées, éventuellement, dans d'autres écoles de droit, dans les instituts des avocats et aux réunions de certaines associations, comme l'Union internationale des avocats, au sein de laquelle le Brésil a plusieurs membres actifs, qui présentent leurs rapports et travaux devant les diverses commissions. Un des congrès de cette institution s'est notamment tenu au Brésil, sous la présidence d'un brésilien, le professeur Arnoldo Medeiros da Fonseca.

Une tentative de rapprochement avec les universités américaines a été effectuée, en 1965, avec l'organisation d'un cours donné par des Professeurs brésiliens et américains, utilisant en grande partie le case method, en s'inspirant des techniques d'enseignement du droit des facultés américaines. Cette initiative, qui a obtenu l'appui de la Fondation Ford et des Universités de Harvard, Yale et Columbia, ainsi que de la Fondation Getulio Vargas et de l'Université de Rio de Janeiro, a exercé une importante influence dans le sens, d'une part, de stimuler l'intérêt des juristes américains pour le droit brésilien et, d'autre part, de modifier la forme traditionnelle de l'enseignement brésilien du droit. ${ }^{64}$ Plusieurs ouvrages ont été publiés, tant au Brésil qu'aux Etats Unis, donnant suite à cette forme de collaboration entre les juristes des deux pays, qui malheureusement n'a duré que quelques années. ${ }^{65}$

Récemment, plusieurs instituts culturels se sont spécialisés dans le droit du Mercosul, en rapprochant les juristes brésiliens et argentins, pour discuter des problèmes juridiques qui intéressent les deux pays et le Mercosul.

En ce qui concerne la bibliographie juridique brésilienne de droit comparé, nous avons, outre certains ouvrages déjà cités de la fin du XIXème siècles, quelques livres de droit civil et de droit public comparé, publiés soit en portugais, soit en français, soit en anglais et même en allemand.

Il faut également noter que ces dernières années, une centaine d'articles sur le droit étranger et le droit comparé ont été publiés dans des revues générales de droit au Brésil. De même, plusieurs articles sur le droit brésilien ont été publiés à l'étranger, en français, en anglais, en italien et en allemand, dans des revues de droit comparé ou dans autres périodiques, spécialisés ou non

Un intérêt croissant pour le droit étranger et le droit comparé se fait sentir de la part non seulement des universités, mais aussi des associations d'avocats et du Conseil Fédéral de l'Ordre et de certains Conseils locaux, comme celui de São Paulo.

Des accords avec des universités étrangères et des échanges de professeurs s'organisent de manière régulière ou ponctuelle avec le Portugal, la France, l'Italie et l'Espagne, de même qu'un nombre croissant de stagiaires américains ou européens viennent passer un certain

$\overline{64}$ Arnoldo WALD, O ensino jurídico: análise e reforma, publication des Travaux du Séminaire sur l'enseignement juridique réalisé par l'Institut des Avocats Brésiliens, en 1969 et James a. GARDNER; Legal imperialism, Madison, The University of Wisconsin Press, 1980. ${ }_{65}$ Parmi d'autres, par son originalité, doit être cité le livre de Keith S. ROSENN; O jeito na cultura jurídica brasileira, avec la préface de Arnoldo WALD. Rio de Janeiro: Renovar, 1998. 
temps au Brésil, et à l'inverse des cabinets brésiliens s'implantent à l'étranger ou intègrent des groupes internationaux d'avocats.

Finalement, les tribunaux brésiliens et les écoles de la magistrature multiplient leurs contacts avec l'étranger, pour des études scientifiques ou à fin d'organiser une collaboration institutionnelle.

On assiste, au fond, à une nouvelle phase pour le Brésil, dépassant l'improvisation et le travail individuel de quelques uns pour faire du droit comparé une branche institutionnelle des études juridiques et une méthode de travail, avec des institutions réellement spécialisées, en collaboration avec les diverses professions intéressées (avocats, magistrats, professeurs et membres du Ministère Public) et des contacts et des relations suivies avec l'extérieur.

Si l'on peut dire du juriste brésilien, à l'instar de son homologue canadien, que la vocation du droit comparé est dans ses gènes ${ }^{66}$, il convient maintenant de coordonner et d'institutionnaliser, au sein de l'enseignement du droit, la connaissance des grands systèmes de droit étranger et d'initier avocats et magistrats au droit comparé et étranger. A cet effet, il sera nécessaire, dans le but de concentrer d'une manière pertinente les efforts et ressources pour obtenir les meilleurs résultats, d'organiser, d'intensifier et de rationaliser le travail fait au Brésil et la collaboration internationale.

Cet effort doit se réaliser sur le plan de l'enseignement universitaire et de la formation professionnelle des juges, des avocats et du Ministère Public.

Il a comme but la connaissance du droit étranger, de la méthode comparative et l'amélioration du droit national, mais il signifie également une vraie ouverture vers l'extérieur et une nouvelle vision du juriste en ce qui concerne la compréhension entre les peuples et le partenariat entre les Etats sur le plan international et régional.

Ainsi, à coté des intérêts professionnels et universitaires et de l'harmonisation des législations régionales, le juriste brésilien devra suivre, dans cette nouvelle phase de mondialisation, l'exemple des comparatistes européens et en particulier de René David, qui a beaucoup étudié notre pays qu'il a fini par très bien connaître et apprécié,

${ }_{66}$ Discours de Mme le Juge Claire L'Heureux-Dubé, Présidente du XIIIème Congrès Internationale de Droit Comparé, prononcé le 24 août 1999, In: Académie Internationale de Droit Comparé, Rapports Généraux, éd. Les Editions Yvan Blais Inc., 1992, p. XXIX. dont le Professeur André Tunc a eu l'occasion d'écrire:

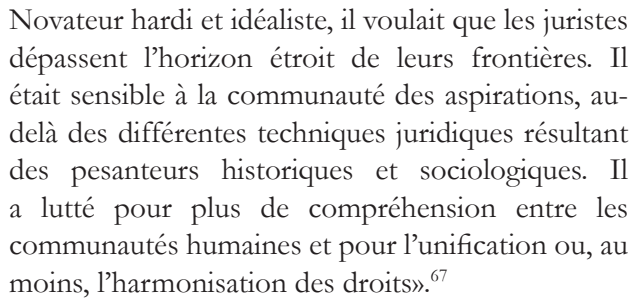

\section{Conclusion}

On a vu tout au long de cet article que, por des raisons de nature historique et culturelle, le Droit Comparé jouait un rôle fondamental por le Droit brésilien, en tant qu'instrument de son développement et de sa conformation comme un ordre juridique autonome, encore au XIXème siècle. Paradoxalement, toutefois, au cours du XXème siècle jusqu'à nos jours, le Droit Comparé a eu une projection et une influence limitées, timides, tant dans la tradition de l'éducation juridique universitaire que dans la pratique des avocats ou même des juges. Cela met en évidence la nécessité d'approfondir et d'encourager l'utilisation du Droit Comparé, un défi qui se présente comme un objectif très salutaire, vu qu'il enrichira la culture juridique brésilienne et sera en outre pleinement conforme à la vocation propre du Droit brésilien, particulièrement au début du XXIème siècle: celle de son internationalisation.

\section{Bibliographie}

ASCARELLI;Tullio. Studi di Diritto Comparato. Milano: Giuffrè, 1952.

AZULAY, Fortunato. Os Fundamentos do Direito Comparado. Rio de Janeiro: Edição da Empresa a Noite, 1950.

BASTIDE, Roger. Brésil: terre des contrastes. Paris: Librairie Armand Colin, 1959.

BEVILAQUA Clóvis. Lições de Legislação Comparada. 2. ed. Bahia: José Luiz da Fonseca Magalhães Editor, 1897.

BLANC-JOUVAN, Xavier. L'influence du Code civil sur les codifications étrangères récentes. In: $\mathrm{Le}$ Code $\mathrm{Ci}$ vil (1804-2004) Livre du bicentenaire. Paris: Dalloz-Litec,

\footnotetext{
67 André TUNC, Jalons dits et écrits. Paris: Société de législation com-
} parée, 1991. p. 43. 
2004. p. 483.

BLANC-JOUVAN, Xavier. " La formation au droit comparé, le point de vue d'un universitaire ». Revue internationale de droit comparé, 1996.

CANIVET, Guy. Tirer profit du droit brésilien. In: WALD, Arnoldo; JAUFFRET-SPINOSI, Camille (Dir.), Le droit brésilien d'bier, d'aujourd'bui et de demain; $\mathrm{Pa}$ ris: Société de Législation Comparée, 2005.

CHARVIN, Robert. L'Investissement International et le Droit au Développement. Paris: L'Harmattan, 2002.

DANTAS, Ivo. Direito Comparado como ciência. Revista de Informação Legislativa, Brasília, n.134, p. 231, 1997.

DAVID, René. Le droit brésilien jusqu'en 1950. In: WALD, Arnoldo; JAUFFRET-SPINOSI, Camille (Dir.). Le droit brésilien d'bier, d'aujourd'bui et de demain; $\mathrm{Pa}$ ris: Société de Législation Comparée, 2005.

DAVID, René. Traité élémentaire de Droit Civil Comparé. Paris: LGDJ, 1950.

DAVID, René; JAUFFRET-SPINOSI, Camille. Les grands systèmes de droit contemporains. Paris: Dalloz, 1992.

DEFFAINS, Bruno (Dir.). L'Analyse Économique du Droit dans les Pays de Droit Civil. Paris: Éditions Cujas, 2002.

DELGADO, Ana Paula Texeira. O Direito ao Desenvolvimento na Perspectiva da Globalização: paradoxes e desafios. Rio de Janeiro: Renovar, 2001.

DORNBUSCH, Rudi. Vérifier la législation avant d'investir. Gazeta Mercantil, 28 août 1996.

ERIK, Jayme. Visões para uma teoria pós-moderna do direito comparado. Revista dos Tribunais, São Paulo, n. 759, 1999.

FAORO, Raymundo. Os donos do Poder. Rio de Janeiro: Globo, 1958.

FAUVARQUE-COSSON, Bénédicte. Le droit brésilien, cet "inconnu" qui ne l'est plus. In: CAMPOS, Diogo Leite de; MENDES, Gilmar Ferreira; MARTINS, Ives Gandra da Silva (Coord.). A evolução do direito no século XXI: estudos em homenagem ao Prof. Arnoldo Wald. Coimbra: Almedina, 2007.

FREYRE, Gilberto. Casa Grande \& Senzala. Rio de Janeiro: Maia e Schmidt Ltda, 1933.

FROMONT, Michel; FRISON-ROCHE, Marie-Anne; COSTA, Thales Morais da; CERQUEIRA, Gustavo; GRAEFF, Bibiana; VILARIÑO, Tanísia martini (co- ord.). Direito francês e direito brasileiro: perspectivas nacionais e comparadas. São Paulo: IDP-Saraiva, 2017.

GARDNER, James A. Legal imperialism. Madison: The University of Wisconsin Press, 1980.

GUSMÃO, Paulo Dourado de. Direito Comparado, sua realidade e suas utopias. Revista Forense, v. 152, p. 20, 1954.

GUSMÃO, Paulo Dourado de. Filosofia do direito e direito comparado. Revista Forense, v. 146, p. 3, 1953.

L'HEUREUX-DUBE, Claire. Discours. In: XIIIème Congrès Internationale de Droit Comparé, prononcé le 24 août 1999, Académie Internationale de Droit Comparé, Rapports Généraux, Quebec, Édition Yvon Blais, 1992.

HOLANDA, Sérgio Buarque de. Raízes do Brasil. Rio de Janeiro: José Olympio Ed., 1936.

JAUFFRET-SPINOSI, Camille. La circulation du modèle juridique français: rapport des journées francoitaliennes de l'Association Henri Capitant de 1993, Travaux de l'Association, Tome XLIV. Paris: Litec, 1994.

KELSEN, Hans. Teoria Pura do Direito. São Paulo: Martins Fontes, 1998.

LAMBERT, Jacques. Le Brésil. Paris: Armand Colin, 1953.

LAMBERT, Jacques. Os Dois Brasis (Les deux Brésils). 12. ed. São Paulo: Editora Nacional, 1984.

LEAL, Victor Nunes. Coronelismo, enxada e voto. São Paulo: Alfa-Ômega, 1975.

LEME, Lino Morais. Direito Civil Comparado. São Paulo: Revista dos Tribunais, 1962.

LINOTTE, D.; MESTRE, A. Services publics et droit public de l'economie. Paris: Litec, 1982.

MELLO, Celso D. de Albuquerque. Direito Internacional Econômico. Rio de Janeiro: Renovar, 1993.

MONTEIRO, João. A Universalização do Direito. São Paulo, 1982.

MORAZE, Charles. Les trois âges du Brésil. Paris: Librairie Armand Colin, 1954.

NADELMAN, Kurt H. Studi in memoria di Tullio Ascarelli. In: Great Comparative Lanyers. Milan: Giuffrè, 1969, vol. 3, p. 1410.

OLIVEIRA, Candido de. Curso de Legislação Comparada. 
Rio de Janeiro: Jacinto Ribeiro dos Santos Editor, 1903.

PALMER, Vernon Valentine. Mixed Jurisdiction Worldwide: the Third Legal Family. Cambridge: University Press, 2001.

PERSPECTIVES du Droit Économique: dialogues avec Michel Jeantin. Paris: Dalloz, 1999.

PERRONE-MOISES, Cláudia. Direito ao Desenvolvimento e Investimentos Estrangeiros. São Paulo: Ed. Oliveira Mendes, 1998.

POSNER, Richard A. Frontiers of Legal Theory. Cambridge: Harvard University Press, 2001.

POSNER, Richard A. Overcoming Law. Cambridge: Harvard University Press, 1995.

ROSENN, Keith S. O jeito na cultura juridica brasileira. Prefácio de Arnoldo Wald. Rio de Janeiro: Renovar, 1998.

SACCO, Rodolfo. La comparaison juridique au service de la connaissance du droit. Paris: Economica, 1991.

SANTOS, Claudio. L'influence du droit français sur la jurisprudence du Supérieur Tribunal de Justice, Rapport des Journées franco-italiennes de l'Association Henri Capitant, Travaux de l'Association Henri Capitant, Tome XLIV. Paris: Litec, 1994.

SERVOIN, François. Droit Administratif de l'Économie. 2. ed. Grenoble: Presses Universitaires de Grenoble, 2001.

TUNC, André. Jalons: Dits et écrits. Paris: Société de Législation Comparée, 1991.

VALCKE, Cahterine. Comparationg law. Compartive law as reconstruction of collective commitments. Cambridge: University Perss, 2018.

WALD, Arnoldo. La Circulation du Modèle Français en Amerique Latine. In: Travaux de l'Association Henri Capitant pour la Culture Juridique Française - La circulacion du modele juridique français au Bresil, Journées franco-italiennes, 1993. Paris: Litec, 1994.

WALD, Arnoldo. Il Diritto dell'Economia e il Diritto dello Svilupo in Brasile. In: Diritto dei nuovi mondi. Atti del convegno promosso dall'Instituto di Diritto Privato delle Facoltá di Giurisprudenza. 5-7 nov. 1992, Genova: CEDAM, 1994.

WALD, Arnoldo, ROCHA, Roberto Paraíso (org.). O ensino jurídico, análise e reforma. Rio de Janeiro: Instituto dos Advogados do Brasil, 1969.
WALD, Arnoldo. La réforme du droit brésilien. Revue internationale de droit comparé, n. 4, p. 713, 1962.

WINIZKY, Ignacio. De la technique comparative en droit. In: Problèmes contemporains de droit compare. Paris: Institut Japonais de Droit Comparé, Université Chuo, Tome II, 1962.

WITZ, Claude. L'influence des codifications nouvelles sur le Code Civil. In: 1804-2004 Le Code civil, un Passé, un Présent, un Avenir. Paris: Dalloz, 2004. 
Para publicar na Revista de Direito Internacional, acesse o endereço eletrônico www.rdi.uniceub.br ou www.brazilianjournal.org.

Observe as normas de publicação, para facilitar e agilizar o trabalho de edição. 